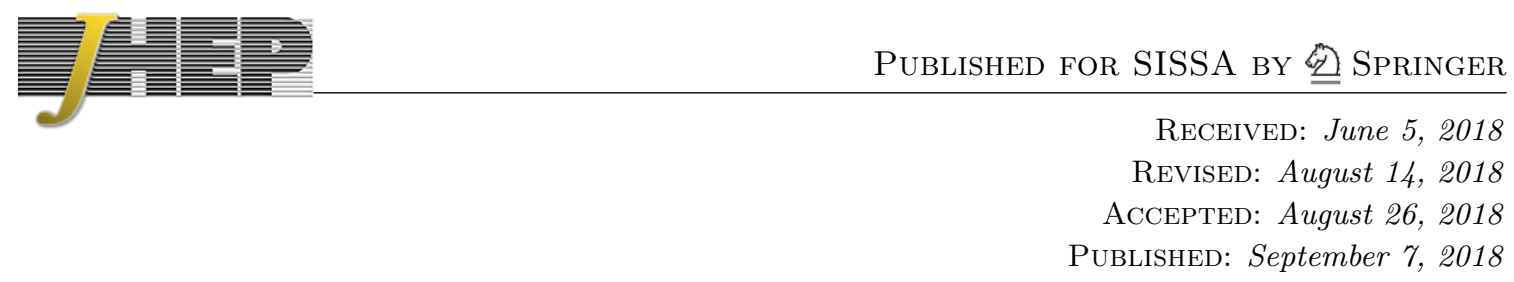

\title{
Logarithmic accuracy of parton showers: a fixed-order study
}

\author{
Mrinal Dasgupta, ${ }^{a}$ Frédéric A. Dreyer, ${ }^{b}$ Keith Hamilton, ${ }^{c, d}$ Pier Francesco Monni ${ }^{d}$ \\ and Gavin P. Salam ${ }^{d, 1}$ \\ ${ }^{a}$ Consortium for Fundamental Physics, School of Physics and Astronomy, \\ University of Manchester, \\ Manchester M13 9PL, U.K. \\ ${ }^{b}$ Center for Theoretical Physics, Massachusetts Institute of Technology, \\ Cambridge, MA 02139, U.S.A. \\ ${ }^{c}$ Department of Physics and Astronomy, University College London, \\ London, WC1E 6BT, U.K. \\ ${ }^{d}$ Theoretical Physics Department, CERN, \\ CH-1211 Geneva 23, Switzerland \\ E-mail: Mrinal.Dasgupta@manchester.ac.uk, fdreyer@mit.edu, \\ keith.hamilton@ucl.ac.uk, pier.monni@cern.ch, gavin.salam@cern.ch
}

ABSTRACT: We formulate some first fundamental elements of an approach for assessing the logarithmic accuracy of parton-shower algorithms based on two broad criteria: their ability to reproduce the singularity structure of multi-parton matrix elements, and their ability to reproduce logarithmic resummation results. We illustrate our approach by considering properties of two transverse-momentum ordered final-state showers, examining features up to second order in the strong coupling. In particular we identify regions where they fail to reproduce the known singular limits of matrix elements. The characteristics of the shower that are responsible for this also affect the logarithmic resummation accuracies of the shower, both in terms of leading (double) logarithms at subleading $N_{C}$ and next-to-leading (single) logarithms at leading $N_{C}$.

KEYwORDS: NLO Computations, QCD Phenomenology

ARXIV EPRINT: 1805.09327

\footnotetext{
${ }^{1}$ On leave from CNRS, UMR 7589, LPTHE, F-75005, Paris, France and from Rudolf Peierls Centre for Theoretical Physics, 1 Keble Road, Oxford OX1 3NP, U.K. .
} 


\section{Contents}

1 Introduction 1

2 Parton showering and our choice of algorithms 4

2.1 Pythia $p_{t}$-ordered shower 5

$\begin{array}{lll}2.2 & \text { Dire shower } & 7\end{array}$

3 Singularity structure of resulting matrix elements $\quad 8$

$\begin{array}{ll}3.1 \text { Single-emission case } & 9\end{array}$

$\begin{array}{lll}3.2 & \text { Issues in two-emission case: double strong ordering } & 12\end{array}$

$\begin{array}{lll}3.3 & \text { Issues in two-emission case: single strong ordering } & 15\end{array}$

4 Logarithmic analysis at second order $\quad 17$

$\begin{array}{lll}4.1 & \text { Subleading- } N_{\mathrm{C}} \alpha_{s}^{2} L^{4} \text { terms } & 18\end{array}$

$\begin{array}{lll}4.2 & \text { Leading- } N_{\mathrm{C}} \alpha_{s}^{2} L^{2} \text { terms } & 20\end{array}$

5 Conclusions $\quad 22$

A Evaluation of double-soft effective matrix element 24

B Fixed-order difference with respect to NLL resummation 25

\section{Introduction}

One of the most central and flexible tools in collider physics is the general purpose Monte Carlo (GPMC) event generator [1], which simulates fully realistic collider events. Event generators involve various components to simulate physics at a range of different scales: for example "hard" scattering matrix elements describe the physics occurring at the electroweak and $\mathrm{TeV}$ scales, the physics that colliders are mainly built to probe. Hadronisation models simulate the GeV-scale ("soft") processes by which quarks and gluons transform from and to the hadrons that are collided and detected. Parton shower algorithms, the subject of this article, provide the crucial connection between the hard and soft scales, simulating mostly strong-interaction physics across the two, three or even more orders of magnitude of momenta that separate them.

GPMCs are extremely successful programs, able to reproduce much of the data from CERN's Large Hadron Collider and its predecessors, sometimes to quite high accuracy. Part of this success is thanks to substantial progress in the past 20 years in improving the description of the hard scale, for example with simultaneous matching to multiple tree-level matrix elements [2-4]; inclusion of next-to-leading (NLO) order corrections through the MC@NLO [5, 6] and POWHEG [7-9] and other [10] methods; and more recently merging 
of NLO corrections across processes of varying jet multiplicity simultaneously [11-13], and the incorporation of NNLO corrections in colour-singlet production simulations [14-16]. Another important area of development contributing to the success of today's GPMCs has been in their non-perturbative models, for example for multiple interactions [17-25], as well as their tuning to data $[26,27]$.

As GPMCs evolve towards higher accuracies, a number of factors make it increasingly important to re-examine their parton shower component. For example: (1) the ability to match NNLO and higher-order calculations with parton showers is to some extent limited by the fact that parton showers do not reproduce the known structure of singularities that is present in a NNLO calculation. This is an issue especially for extensions of the MC@NLO method. (2) The parton shower provides the initial conditions for hadronisation, and the quality of a tune of hadronisation models can well be affected by the quality of that initial condition. This is especially true if one tunes the model predominantly using data at one energy scale (e.g. LEP energies) and wishes to use the same tune for much higher-energy processes (e.g. multi-TeV processes at the LHC), where the parton shower is effectively providing much of the extrapolation. (3) There is an increasing trend towards exploiting information over the full range of momenta between the hard scale and the hadronisation scale, notably in jet substructure studies, using both hand-crafted observables and machine learning [28]. Parton showers are the only tool with the flexibility to predict the relevant dynamics for arbitrarily complex observables across that range of scales. (4) A number of experimental measurements are becoming limited by systematic errors that have their origins in differences between GPMCs, and one cannot help but wonder whether a better understanding of parton showers could resolve this situation. A notable example is the jetenergy-scale systematic uncertainty, for which differences in quark and gluon fragmentation between different GPMCs contribute significantly [29, 30].

There are several ongoing efforts to improve QCD parton showers, which can be classified into three broad kinds of activity: (1) developing novel showers that remain within the standard $1 \rightarrow 2$ or $2 \rightarrow 3$ branching paradigms, for example the Dire [31], Vincia [32, 33] or Deductor [34] showers. (2) Incorporating more information about interferences, particularly relevant for spin and colour degrees of freedom, e.g. refs. [34-38]. (3) Incorporating higher-order splitting functions [10, 39-44]. These efforts have brought significant innovations, however there remains a need for a broader, systematic framework in which to think about the question of parton-shower accuracy, so as to help frame and guide such developments. In particular to evaluate the advances brought by any single improvement to a shower (e.g. higher-order splitting functions), one needs to understand its interplay with the shower as a whole. It is therefore the purpose of this article to sketch such a framework and draw some first conclusions within it.

An important consideration in discussing the accuracy of parton showers is that they may be used to calculate essentially any observable. This is to be contrasted with the situation for fixed order calculations, where one selects a given observable, e.g. $n$-jet kinematic distributions, and immediately knows which inputs are needed for a given perturbative accuracy. Here we suggest that a framework for discussing parton shower accuracy should involve at least two core elements. 
The first element of our proposed framework reflects the fact that parton showers effectively generate an approximation to the tree-level $n$-parton matrix elements for any $n$. One can ask in what sense that approximation is correct. Typically one would expect the parton shower to reproduce the matrix element in a variety of ordered limits, where one or other kinematic property of emissions is ordered from one emission to the next, e.g. $p_{\perp, n} \ll p_{\perp, n-1} \ll \ldots \ll p_{\perp, 1} \ll Q$ (here $p_{\perp, i}$ is the transverse momentum of particle $i$ and $Q$ is the centre-of-mass energy). Much is known about the properties of matrix elements in such ordered limits, e.g. refs. [45-48].

We note that early, pioneering work on transverse-momentum ordered showers with dipole-local recoil $[49,50],{ }^{1}$ did consider comparisons to matrix elements, both doubleemission matrix elements [51] and, from the point of view of the colour structure [52], multi-parton matrix elements [53, 54]. The lessons and algorithms derived from ref. [51], about recoil prescriptions, were included in the Ariadne dipole shower [50], while those from ref. [53] were incorporated in a modification used in ref. [54]. We further discuss these articles below.

The second element of our framework makes a connection with resummation. Resummation accounts for logarithmically enhanced terms $\alpha_{s}^{n} L^{m}$ to all orders, where $\alpha_{s}$ is the strong coupling and $L$ is the logarithm of the ratio of any two physical scales. Resummations are classified according to the specific set of dominant and subdominant terms that are under control. It is natural to ask what resummation accuracy will be obtained within a given shower, for each observable where resummed results exist. While this appears to be an observable-specific question, resummations exist for large classes of observables [55, 56] and so in effect one can ask questions about parton-shower resummation accuracy across all observables within those classes. Note that early work on showers [57] gave particular emphasis to the question of the scaling of particle multiplicities with centre-of-mass energy, which is calculable analytically.

There are natural connections between the two elements of our framework. For example, a failure to reproduce appropriate matrix-element limits often occurs together with a failure to obtain a related resummation accuracy for certain observables. Nevertheless the two elements are also complementary: on one hand the matrix-element conditions are relevant for observables of arbitrary complexity, for which no resummation exists; on the other hand the resummation conditions more immediately constrain aspects associated with non-trivial virtual corrections, for example the scale of the strong coupling. The two elements are not necessarily exhaustive in terms of the types of requirements one may ask of showers, but as we will see they are already informative and constraining.

To make our discussion concrete, it will be helpful to examine the case of specific showers. We will consider two transverse-momentum ordered showers with dipole-local recoil: the Pythia shower [21], and the Dire shower [31], restricting our attention to massless final-state splittings. The algorithms underlying these showers are described in section 2 . Then in section 3 we will outline how to usefully classify the ability of showers

\footnotetext{
${ }^{1}$ The shorthand term "dipole shower" appears to have different meanings for different groups, and so we avoid its use.
} 
to reproduce $n$-parton matrix elements. This will reveal issues already at the two-emission level. Section 4 will then show how these issues connect with the question of logarithmic resummation accuracy. Again a second-order analysis will be sufficient to highlight the main features.

\section{Parton showering and our choice of algorithms}

There are many parton showers being used and under further development today. They generate emissions in a sequence according to a kinematic ordering variable. One way of classifying showers is based on their specific choice of ordering variable. The most common choice is to order emissions in transverse momentum and all three major Monte Carlo programs have such a shower: Pythia [58, 59] uses the shower of ref. [21], Sherpa [60] uses the shower of ref. [61] and Herwig has as an option the shower of ref. [62]. Other transverse-momentum showers include Vincia [32,33], available in Pythia, and Dire [31], available for both Pythia and Sherpa. Another class of shower orders emissions in angle [57,63, 64] and is used mainly in the Herwig Monte Carlo programs [65]. Finally there is the Deductor shower work (ref. [34] and references therein), which considers an ordering variable that is related to emission time, but also discusses transverse-momentum ordering. Its code is standalone.

A further important distinguishing feature of each parton shower is the way in which the recoil associated with the emitted parton at a given evolution step is absorbed by other particles in the event. Some shower algorithms rely on a local scheme, in which the recoil is shared among the two colour-connected partners of the emission. Another approach is to use a global scheme, which distributes the recoil among all other particles in the event.

It is beyond the scope of this article to consider all of these showers. We rather choose to concentrate on two of them: (1) the Pythia shower on the grounds that it is today's most extensively used shower; and (2) the Dire shower [31], on the grounds that it is the only shower explicitly available in two Monte Carlo simulation programs (Pythia [59] and Sherpa [60]) and that it is being used as a basis for the inclusion of higher-order splitting kernels [41-43]. Both are transverse-momentum ordered and use recoil that is kept local within colour dipoles. ${ }^{2}$

To help our discussion it is useful to give a summary of the ingredients of common parton shower algorithms. We use $\mathcal{S}_{n}$ to denote a specific kinematic state with $n$ partons. The probability $P\left(\mathcal{S}_{n}, v\right)$ of finding that state is a function of a kinematic ordering variable $v$. A first key component of a parton shower algorithm is a differential equation for the evolution of that probability as the ordering variable is decreased:

$$
\frac{d P\left(\mathcal{S}_{n}, v\right)}{d \ln 1 / v}=-f\left(\mathcal{S}_{n}, v\right) P\left(\mathcal{S}_{n}, v\right) .
$$

The second component of the parton shower algorithm is a kinematic mapping from the state $\mathcal{S}_{n}$ to an $n+1$-particle state $\mathcal{S}_{n+1}$. The map is a function of the ordering variable,

\footnotetext{
${ }^{2}$ Neither of these showers claims NLL accuracy. For example the Pythia manual states "While the final product is still not certified fully to comply with a NLO/NLL standard, it is well above the level of an unsophisticated LO/LL analytic calculation." [58].
} 
the choice of the partons involved in the branching and two additional kinematic variables which we call $z$ and $\phi$. Insofar as we deal with showers with recoil that is local to the colour dipole that is splitting, one should choose two partons, which we label $i$ and $j$. We write the mapping as

$$
\mathcal{S}_{n+1}=\mathcal{M}\left(\mathcal{S}_{n}, v ; i, j, z, \phi\right) .
$$

This kinematic map has an associated "splitting" weight function $d \mathcal{P}\left(\mathcal{S}_{n}, v ; i, j, z, \phi\right)$, which governs the relative probabilities of the different possible new states and which can be conveniently normalised so as to relate it to the $f\left(\mathcal{S}_{n}, v\right)$ function of eq. (2.1),

$$
f\left(\mathcal{S}_{n}, v\right)=\sum_{i, j} \int d v^{\prime} d z d \phi \frac{d \mathcal{P}\left(\mathcal{S}_{n}, v^{\prime} ; i, j, z, \phi\right)}{d v^{\prime} d z d \phi} \delta\left(\ln v^{\prime} / v\right) .
$$

Eq. (2.1) encodes the virtual contributions associated with maintaining the system in state $\mathcal{S}_{n}$. Eq. (2.3) states that virtual and real contributions should be equal (aside the opposite sign), i.e. that probability is conserved, which is also referred to as unitarity. In suitable soft and/or collinear limits (we return to this in section 3) one expects the splitting weight function to be closely related to the ratio of $n+1$ and $n$-parton matrix elements and phase space. Schematically, one might write this as

$$
\sum_{i, j} d \mathcal{P}\left(\mathcal{S}_{n}, v ; i, j, z, \phi\right) \simeq \frac{d \Phi_{n+1}}{d \Phi_{n}} \frac{\left|M^{2}\left(\mathcal{S}_{n+1}\right)\right|}{\left|M^{2}\left(\mathcal{S}_{n}\right)\right|} .
$$

For typical dipole showers, the sum over $i$ runs over all emitting particles and $j$ over all colour connected partners, at most two for each $i$ at leading colour. Colour factors and the relevant factor of the strong coupling are included in $d \mathcal{P}$.

The difference between one shower and another lies not just in the choice of kinematic ordering variable $v$, but also in the mapping function $\mathcal{M}$ and the splitting weight function $\mathcal{P}$. For a given colour dipole $i j$, the showers that we consider here separate the phase space into a region that is predominantly collinear to $i$ and another that is predominantly collinear to $j$.

Note that for the purpose of this article we will only consider final-state showers, with massless partons.

\subsection{Pythia $p_{t}$-ordered shower}

Pythia's transverse-momentum ordered shower [21], is the default option of the Pythia8 program [59] and was also available in Pythia6 [58]. The exposition that follows, restricted to its final-state branching elements, is based on ref. [21] and inspection of the Pythia8 code, version 8.266.

The ordering variable $v$ is a transverse momentum, which is referred to as $p_{\perp, \mathrm{evol}}$,

$$
v \equiv p_{\perp, \mathrm{evol}} .
$$

The map $\mathcal{M}\left(\mathcal{S}_{n}, v ; i, j, z, \phi\right)$ takes massless pre-branching momenta $\widetilde{p}_{i}$ and $\widetilde{p}_{j}$ and constructs post branching momenta $p_{i}, p_{j}$ and $p_{k}$, corresponding to a branching $\tilde{p}_{i} \rightarrow p_{i}+p_{k}$ with 
spectator particle $j$ taking longitudinal recoil to ensure momentum conservation. It is useful to define intermediate variables

$$
\rho_{\perp, \mathrm{evol}}^{2}=\frac{p_{\perp, \mathrm{evol}}^{2}}{\left(\widetilde{p}_{i}+\widetilde{p}_{j}\right)^{2}}, \quad y=\frac{\rho_{\perp, \mathrm{evol}}^{2}}{z(1-z)}, \quad \tilde{z}=\frac{(1-z)\left(z^{2}-\rho_{\perp \mathrm{evol}}^{2}\right)}{z(1-z)-\rho_{\perp \mathrm{evol}}^{2}},
$$

and the Catani-Seymour [66] style dipole map is then defined by

$$
\begin{aligned}
p_{i}^{\mu} & =\widetilde{z} \widetilde{p}_{i}^{\mu}+y(1-\widetilde{z}) \widetilde{p}_{j}^{\mu}+k_{\perp}^{\mu}, \\
p_{k}^{\mu} & =(1-\widetilde{z}) \widetilde{p}_{i}^{\mu}+y \widetilde{z} \widetilde{p}_{j}^{\mu}-k_{\perp}^{\mu}, \\
p_{j}^{\mu} & =(1-y) \widetilde{p}_{j}^{\mu} .
\end{aligned}
$$

Here $k_{\perp}^{\mu}$ is defined as

$$
k_{\perp}^{\mu}=\sqrt{\widetilde{z}(1-\widetilde{z}) y\left(\widetilde{p}_{i}+\widetilde{p}_{j}\right)^{2}}\left[\widehat{k}_{\perp, 1}^{\mu} \cos \phi+\widehat{k}_{\perp, 2}^{\mu} \sin \phi\right],
$$

where $\widehat{k}_{\perp, 1}^{\mu}$ and $\widehat{k}_{\perp, 2}^{\mu}$ are four-vectors that are orthogonal to each other as well as to $\widetilde{p}_{i}^{\mu}, \widetilde{p}_{j}^{\mu}$, and that satisfy $\widehat{k}_{\perp, 1}^{2}=\widehat{k}_{\perp, 2}^{2}=-1$. Eqs. (2.7)-(2.8) imply

$$
\left|k_{\perp}^{2}\right|=\frac{\left(z^{2}-\rho_{\perp \text { evol }}^{2}\right)\left((1-z)^{2}-\rho_{\perp \text { evol }}^{2}\right)}{\left(z(1-z)-\rho_{\perp \text { evol }}^{2}\right)^{2}} p_{\perp, \mathrm{evol}}^{2} .
$$

The squared transverse momentum $\left|k_{\perp}^{2}\right|$ that is assigned to the emission coincides with the ordering variable $p_{\perp, \text { evol }}^{2}$ when $z,(1-z) \gg \rho_{\perp, \text { evol }}$, i.e. in the collinear limit. The map only exists for

$$
\rho_{\perp, \mathrm{evol}} \leq z \leq 1-\rho_{\perp, \mathrm{evol}},
$$

and, at the edges of this range, $\left|k_{\perp}\right|$ vanishes even for finite $p_{\perp, \text { evol }}$.

If parton $i$ is a quark, only a $q \rightarrow q g$ branching is possible, and the quark is colour connected to only one other particle in the event (the spectator $j$ ). The splitting weight function in this case is given by

$$
d \mathcal{P}_{q \rightarrow q g}=\frac{\alpha_{s}\left(p_{\perp, \mathrm{evol}}^{2}\right)}{2 \pi} \frac{d p_{\perp \mathrm{evol}}^{2}}{p_{\perp \text { evol }}^{2}} d z \frac{d \phi}{2 \pi} C_{F}\left(\frac{1+z^{2}}{1-z}\right) .
$$

Note the use of the evolution variable $p_{\perp \text {,evol }}^{2}$ in the scale of $\alpha_{s}$, rather than the kinematic quantity $\left|k_{\perp}^{2}\right|$. If parton $i$ is a gluon, both $g \rightarrow g g$ and $g \rightarrow q \bar{q}$ branchings are possible and for each of them, the shower takes into account two colour connections, assigning equal weights to each. The $g \rightarrow g g$ splitting weight is

$$
d \mathcal{P}_{g \rightarrow g g}=\frac{\alpha_{s}\left(p_{\perp, \mathrm{evol}}^{2}\right)}{2 \pi} \frac{d p_{\perp \text { evol }}^{2}}{p_{\perp \text { evol }}^{2}} d z \frac{d \phi}{2 \pi} \frac{C_{A}}{2}\left[\frac{1+z^{3}}{1-z}\right] .
$$

The usual $P_{g g}$ splitting function is reconstructed from this, together with its $1 / 2$ ! symmetry factor, when one considers that each gluon splits separately in each of the two dipoles to 
which it belongs ${ }^{3}$ and that for a $\widetilde{p}_{i} \rightarrow p_{i}+p_{k}$ splitting, observables do not distinguish between gluon $p_{i}$ and gluon $p_{k}$, which provides an implicit symmetrisation of $z \leftrightarrow 1-z$. For the $g \rightarrow q \bar{q}$ case, the following is used

$$
d \mathcal{P}_{g \rightarrow q \bar{q}}=\frac{\alpha_{s}\left(p_{\perp, \mathrm{evol}}^{2}\right)}{2 \pi} \frac{d p_{\perp \text { evol }}^{2}}{p_{\perp \text { evol }}^{2}} d z \frac{d \phi}{2 \pi} \frac{n_{f} T_{R}}{2} \mathcal{D}[1-2 \widetilde{z}(1-\widetilde{z})],
$$

where again the gluon splitting occurs with this weight separately in each of the two dipoles to which it belongs. The factor $\mathcal{D}$ is,

$$
\mathcal{D}=(1-x)^{2}(1+x), \quad x \equiv \frac{\left(p_{i}+p_{k}\right)^{2}}{\left(\widetilde{p}_{i}+\widetilde{p}_{j}\right)^{2}}
$$

Such that in the collinear, limit $\mathcal{D}=1$ and $\widetilde{z}=z$. The Pythia shower has the option of using the CMW [67] scheme for the coupling

$$
\alpha_{s}^{\mathrm{CMW}}\left(p_{\perp, \mathrm{evol}}^{2}\right)=\alpha_{s}^{\overline{\mathrm{MS}}}\left(p_{\perp, \mathrm{evol}}^{2}\right)\left(1+\frac{\alpha_{s}^{\overline{\mathrm{MS}}}\left(p_{\perp, \mathrm{evol}}^{2}\right)}{2 \pi} K\right), \quad K=\left(\frac{67}{18}-\frac{\pi^{2}}{6}\right) C_{A}-\frac{10}{9} T_{R} n_{f},
$$

in the soft-enhanced parts of the splitting functions, which is one key element of NLL resummations.

\subsection{Dire shower}

The Dire transverse-momentum ordered shower [31], is available for both the Sherpa [60] and the Pythia8 generation frameworks.

The Dire ordering variable is once again a (squared) transverse momentum type variable and is called $t$,

$$
v \equiv \sqrt{t}
$$

together with the splitting variables $z$ and $\phi$. To construct the final-state kinematic map one defines intermediate variables

$$
\kappa^{2}=\frac{t}{\left(\widetilde{p}_{i}+\widetilde{p}_{j}\right)^{2}}, \quad y=\frac{\kappa^{2}}{1-z}, \quad \widetilde{z}=\frac{z-y}{1-y} .
$$

The Dire map then has the identical form to the Pythia map, i.e. using eqs. (2.7) and (2.8) but with the Dire expressions for $y$ and $\widetilde{z}$. The kinematic squared transverse momentum, expressed in terms of the original splitting variables, is

$$
\left|k_{\perp}^{2}\right|=(1-z) \frac{z(1-z)-\kappa^{2}}{\left(1-z-\kappa^{2}\right)^{2}} t .
$$

In the soft-collinear limit, with $1-z \ll 1$ and $\kappa \ll 1-z$, this reduces to $\left|k_{\perp}^{2}\right|=t$, i.e. the ordering variable is identical to the squared emitted transverse momentum, as in the case

\footnotetext{
${ }^{3}$ I.e. in a $q g \bar{q}$ system, eq. (2.12) applies to the gluon splitting in the $q g$ dipole and the gluon splitting in the $g \bar{q}$ dipole, each of which carries a $C_{A} / 2$ factor.
} 
of the Pythia shower. The limits on $z$ for a given value of $t$ are dictated by the requirement of positivity of the right-hand side of eq. (2.18), and give

$$
\frac{1}{2}-\sqrt{\frac{1}{4}-\kappa^{2}} \leq z \leq \frac{1}{2}+\sqrt{\frac{1}{4}-\kappa^{2}} .
$$

For small $\kappa$, this becomes $\kappa^{2} \leq z \leq 1-\kappa^{2}$. Note that this scales differently from the Pythia case, eq. (2.10), the consequences of which will be discussed below. The Dire splitting weight functions are

$$
\begin{aligned}
& d \mathcal{P}_{q \rightarrow q g}=\frac{\alpha_{s}(t)}{2 \pi} \frac{d t}{t} d z \frac{d \phi}{2 \pi} C_{F}\left[2 \frac{1-z}{(1-z)^{2}+\kappa^{2}}-(1+z)\right] \\
& d \mathcal{P}_{g \rightarrow g g}=\frac{\alpha_{s}(t)}{2 \pi} \frac{d t}{t} d z \frac{d \phi}{2 \pi} \frac{C_{A}}{2}\left[2 \frac{1-z}{(1-z)^{2}+\kappa^{2}}-2+z(1-z)\right] \\
& d \mathcal{P}_{g \rightarrow q \bar{q}}=\frac{\alpha_{s}(t)}{2 \pi} \frac{d t}{t} d z \frac{d \phi}{2 \pi} \frac{n_{f} T_{R}}{2}[1-2 z(1-z)]
\end{aligned}
$$

As in the case of Pythia the gluon splittings apply once for each of the two dipoles to which the gluon belongs. ${ }^{4}$ The Dire shower (like Pythia) has the option of using the CMW scheme in the coupling, i.e. $\alpha_{s}^{\mathrm{CMW}}(t)$. One key difference of Dire relative to the Pythia case is the modification of the soft divergence,

$$
\frac{1}{1-z} \rightarrow \frac{1-z}{(1-z)^{2}+\kappa^{2}}
$$

This introduces an effective cutoff of the soft divergence when $1-z \sim \kappa$, to be contrasted with the actual limit in the kinematic map of $1-z \gtrsim \kappa^{2}$. Thus the Pythia and Dire showers both effectively cut off the divergence for $1-z \sim \kappa$, but Pythia implements this through the kinematic map, while Dire does so through the splitting functions. Physically this cutoff is situated around zero rapidity in a frame in which the dipole is at rest: effectively only one of the dipole's two partons radiates in an $i \rightarrow i+k$ splitting, and the radiation fills the associated hemisphere in the dipole centre of mass.

\section{$3 \quad$ Singularity structure of resulting matrix elements}

For each emission $i$ in an $n$-parton matrix element there are two kinds of singularity, a soft singularity when parton $i$ 's energy goes to zero and a collinear singularity when its angle with respect to any other parton goes to zero. One has considerable freedom in what two variables one uses to describe these two singularities. For example one may use pair-invariant mass and energy, angle and energy, transverse momentum and angle, etc.

The very minimal expectation for a parton shower is that it reproduces the matrix element for any single-emission configuration with one or two singularities: i.e. in the collinear and soft limit, with two singularities; in the collinear and non-soft limit, with one singularity corresponding to the DGLAP splitting functions [68-70]; and in the soft non-collinear limit, reproducing the Bassetto-Ciafaloni-Marchesini eikonal emission formulas [45].

\footnotetext{
${ }^{4}$ The $z$ structure of the $d \mathcal{P}_{g \rightarrow g g}$ formula that we quote is given by the sum of the fsr_qcd_G2GG1: :calc and fsr_qcd_G2GG2: : calc, functions in the Dire 2.001 code. Analogously for the other splitting functions.
} 
Once one considers more than one emission, one reasonable expectation is to control the leading singularity of the squared amplitude for any number of emissions, that is to correctly reproduce the divergence of the matrix element in configurations where each emission triggers two singularities relative to a parent configuration without that emission. For example, the leading singularity for such a configuration might involve two emissions with disparate values both of their transverse momenta and angles. ${ }^{5}$ This is closely connected with the reproduction of leading double logarithms.

Among the elements needed to reproduce subleading-logarithmic corrections, one might also require that when there are one or more emissions that each trigger only one singularity (rather than two), the matrix element is still correctly reproduced. For example, multiple hard emissions that are ordered in angle should reproduce the DGLAP anomalous dimensions (this was discussed and eventually established for dipole type showers in refs. [71-73]). Such a limit also involves spin-correlations, for which an algorithm has long been known [74, 75], though only some showers make use of it.

A potentially delicate configuration with regards to this condition occurs when emissions have commensurate values of the ordering variable, but disparate values of a complementary kinematic variable. For example, in angular-ordered showers, it is known that the matrix element is not correctly reproduced for multiple emissions with commensurate angles but strongly ordered energies. As a result non-global single-logarithmic terms are not correctly reproduced [76].

One can study the reproduction of singularities both exactly and in a leading $N_{\mathrm{C}}$ limit. For example the case of commensurate angles and disparate energies is especially challenging beyond leading $N_{\mathrm{C}}$, even within dedicated resummation approaches, and only one complete answer is known [77].

For what follows, we will work in a limit where all emissions are soft relative to the centre-of-mass energy. They may be strongly ordered both in transverse momentum and angle, or only in the latter. This limit will already prove to be illuminating.

\subsection{Single-emission case}

To reproduce the leading double logarithms, the requirement for the single-emission pattern is that in the limit where an emission has a small energy with respect to the parent system (soft limit) and it has a small angle relative to another parton (collinear limit), the corresponding matrix element times phase space should be reproduced, i.e.

$$
d \mathcal{P}=\frac{2 C \alpha_{s}\left(p_{\perp}^{2}\right)}{\pi} \frac{d p_{\perp}}{p_{\perp}} d \eta
$$

where $C$ is the colour factor of the emitting parton, $\eta$ is a rapidity with respect to the emitting parton and $p_{\perp}$ is a transverse momentum with respect to it. This is straightforwardly reproduced by our selected showers.

For single-logarithmic accuracy, it is essential to also reproduce the emission pattern in the hard collinear region and that in the soft large-angle region. The former is straightfor-

\footnotetext{
${ }^{5}$ For the purposes here, angles are understood to be defined with respect to the more energetic particle to which they are closest in angle.
} 
ward to verify from the equations in section 2, after taking into account the symmetrisation over $z \leftrightarrow(1-z)$ and the fact that gluons radiate separately as part of each of two dipoles. It is therefore the soft large-angle region that we examine here. To discuss the soft largeangle region it is helpful to use (pseudo)rapidity, $\eta=-\ln \tan \theta / 2$ and physical transverse momentum, $\left|p_{\perp}\right|$ of the emission to parametrise its phase space. A variety of definitions can be constructed for the physical transverse momentum, but in the soft limit, for emission from a single colour dipole, all sensible ones will coincide. ${ }^{6}$

For concreteness, consider a $q \bar{q}$ dipole of mass $Q$, in its centre-of-mass frame, with the quark $q$ along the $z$ axis. First we consider branching of the quark. Using eq. (2.7), the emitted gluon has pseudorapidity:

$$
\eta=\ln \frac{(1-\widetilde{z}) Q}{\left|k_{\perp}\right|},
$$

where $k_{\perp}$ in eq. (2.7) coincides with our $p_{\perp}$. The Pythia mapping in the soft limit, $1-z \ll 1$, gives

$$
\text { Pythia: } \quad \eta=\frac{1}{2} \ln \left[\frac{(1-z)^{2}}{\rho_{\perp, \text { evol }}^{2}}-1\right], \quad\left|p_{\perp}^{2}\right|=p_{\perp, \text { evol }}^{2}\left(1-\frac{\rho_{\perp, \text { evol }}^{2}}{(1-z)^{2}}\right) .
$$

For $z / \rho>\sqrt{2}, \eta$ is positive. It is instructive to examine the contour in the $\eta, \ln \left|p_{\perp}\right|$ plane that is covered for a given value of the ordering variable, $v \equiv p_{\perp, \text { evol }}$, together with the splitting function weight differentially along that contour:

$$
\text { Pythia: }\left|p_{\perp}^{2}\right|=p_{\perp, \mathrm{evol}}^{2}\left(\frac{e^{2 \eta}}{1+e^{2 \eta}}\right), \quad d \mathcal{P}_{q \rightarrow q g}=\frac{2 \alpha_{s}\left(p_{\perp, \mathrm{evol}}^{2}\right) C_{F}}{\pi} \frac{d p_{\perp}}{p_{\perp}} d \eta\left(\frac{e^{2 \eta}}{1+e^{2 \eta}}\right),
$$

where we have dropped the $d \phi / 2 \pi$ factor for compactness. For large positive values of $\eta$, $\left|p_{\perp}\right|=p_{\perp, \text { evol }}$ and the splitting weight is independent of $\eta$. For large negative values of $\eta$, $\left|p_{\perp}\right|=e^{-|\eta|} p_{\perp, \text { evol }}$ and the splitting weight is suppressed, but non-zero. The splitting of the anti-quark $\bar{q}$ yields similar results, but with $\eta \rightarrow-\eta$. If one ignores the running of the coupling, the sum of the $q$ and $\bar{q}$ splittings yields

$$
\text { Pythia (ignoring running): } \quad d \mathcal{P}_{q \rightarrow q g}+d \mathcal{P}_{\bar{q} \rightarrow \bar{q} g}=\frac{2 \alpha_{s} C_{F}}{\pi} \frac{d p_{\perp}}{p_{\perp}} d \eta .
$$

This has a uniform distribution in rapidity, which is the correct result for soft-gluon emission from a dipole. The analogue of eqs. (3.3), (3.4) for Dire is

$$
\text { Dire: } \quad \eta=\frac{1}{2} \ln \left[\frac{(1-z)^{2}}{\kappa^{2}}\right], \quad\left|p_{\perp}^{2}\right|=t, \quad d \mathcal{P}_{q \rightarrow q g}=\frac{2 \alpha_{s}(t) C_{F}}{\pi} \frac{d p_{\perp}}{p_{\perp}} d \eta\left(\frac{e^{2 \eta}}{1+e^{2 \eta}}\right) .
$$

Dire and Pythia therefore have identical rapidity distributions for soft emission from one side of a dipole, however in Dire the emission always has $\left|p_{\perp}^{2}\right|=t$, unlike the Pythia case. This means that the sum of quark and anti-quark splittings has a simple weight even taking into account running coupling effects:

$$
\text { Dire: } \quad d \mathcal{P}_{q \rightarrow q g}+d \mathcal{P}_{\bar{q} \rightarrow \bar{q} g}=\frac{2 \alpha_{s}\left(\left|p_{\perp}^{2}\right|\right) C_{F}}{\pi} \frac{d p_{\perp}}{p_{\perp}} d \eta
$$

\footnotetext{
${ }^{6}$ E.g. for a massless emission $p$ from a dipole between massless particles $P_{1}$ and $P_{2}$, one can define $p_{\perp}^{2}=2 \frac{\left(P_{1} \cdot p\right)\left(P_{2} \cdot p\right)}{P_{1} \cdot P_{2}}$.
} 


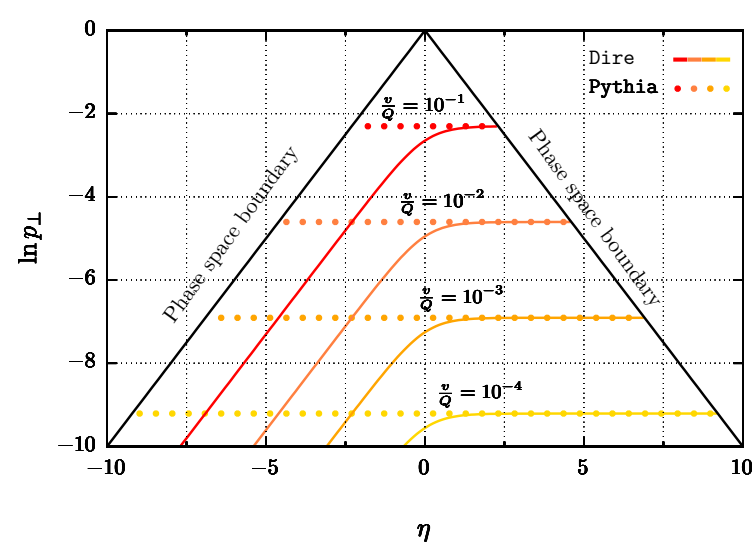

(a)

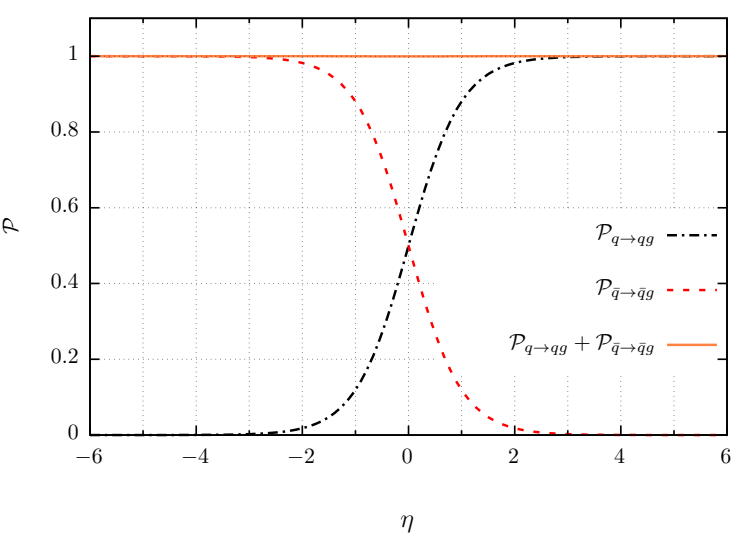

(b)

Figure 1. (a) The accessible contour of emissions in the $\eta-\ln p_{\perp}$ ("Lund" [78]) plane for fixed values of the ordering variable $v$, for splittings of a right-going quark, shown for both the Pythia and Dire shower kernels. The phase-space boundary is sometimes alternatively described as the hardcollinear limit. (b) The splitting weights associated with emissions as a function of rapidity from the (right-going) quark and (left-going) anti-quark, normalised so as to be 1 in the soft-collinear region. This holds for both Pythia and Dire. The weights are independent of $v$, as long as $v / Q \ll e^{-|\eta|}$, i.e. as long as one is far from the phase-space boundary shown in (a). The rapidity is defined in the $q \bar{q}$ dipole centre-of-mass frame.

The essential properties of single-parton emission are illustrated in figure 1. There are three main elements to comment on regarding the above analysis:

1. The effective single-emission matrix element in Pythia and Dire is correct in all singly-divergent regions of phase space, i.e. both soft large-angle and hard-collinear, as well as soft-collinear. In Pythia the invariance of the radiation pattern under boosts along the dipole direction is broken by running coupling effects: the same scale $\mu_{R}=v$ is used along the whole contour of constant $v$, even though, as one sees from figure 1a, that contour maps to a range of different physical $p_{\perp}$ values. This effect is expected to have consequences that are beyond NLL accuracy, because the region where $p_{\perp}$ differs substantially from $v$ comes with a finite weight only at large angle and a strongly suppressed weight in the anti-collinear region, cf. figure 1b. Accordingly we will not discuss it further in this article.

2. In both Pythia and Dire, the dipole is divided into two parts, one associated with the quark, the other with the anti-quark. That division occurs at zero-rapidity in the dipole rest frame, as is visible clearly in figure $1 \mathrm{~b}$. While the sum adds up to one, the two elements of the partition behave differently for subsequent emissions, and the specific choice of partitioning can then have adverse consequences, as we shall see shortly. In particular it will affect subleading- $N_{\mathrm{C}}$ LL terms, and the full set of NLL terms. 
3. Within Pythia, there is a suppressed but non-zero probability to have arbitrarily small kinematic transverse momentum, $\left|p_{\perp}\right|$, for any finite value of the evolution transverse-momentum variable $v \equiv p_{\perp \text {,evol }}$ (i.e. the negative $\eta$ regions of figure 1 for the $q \rightarrow q g$ splitting). This too will have adverse consequences, at LL and leadingcolour accuracy: if one asks what the probability is to have an event whose hardest emission is at some very small scale $p_{\perp, \text { cut }} \ll Q$, normally this implies a Sudakov suppression down to the scale $p_{\perp, \text { cut }}$. However in the Pythia shower there is a second mechanism: full Sudakov suppression down to some intermediate scale $p_{\perp \text {,cut }} \ll$ $p_{\perp, \mathrm{evol}} \ll Q$, together with creation of an emission with $\left|p_{\perp}\right| \sim p_{\perp, \mathrm{cut}} \ll p_{\perp, \mathrm{evol}}$, which comes with a weight suppressed as in eq. (3.4). These two mechanisms compete and for sufficiently small values of $p_{\perp, \text { cut }}$, the second one may dominate, which results in an overall degree of suppression of such configurations which no longer satisfies normal double-logarithmic scaling. In practice, the values of $\ln Q /\left|p_{\perp, \text { cut }}\right|$ at which this occurs are so large that they are unlikely to be of phenomenological interest. Accordingly we will not discuss this point any further here. Nevertheless, in future efforts to design showers, one should be aware that this kind of effect can arise.

\subsection{Issues in two-emission case: double strong ordering}

We now consider double-emission configurations that probe the leading singularity of the double-real squared amplitude, associated with leading (double) logarithms. The region of phase space that we will concentrate on for double emission is that where the two emissions are both soft and collinear to either of the hard partons and widely separated in rapidity from each other, $\left|\eta_{1}-\eta_{2}\right| \gg 1$. In this limit, considering an initial $q \bar{q}$ dipole, the correct double-emission probability has the very simple form

$$
d P_{2}=\frac{C_{F}^{2}}{2 !} \prod_{i=1,2}\left(\frac{2 \alpha_{s}\left(p_{\perp, i}^{2}\right)}{\pi} \frac{d p_{\perp, i}}{p_{\perp, i}} d \eta_{i} \frac{d \phi_{i}}{2 \pi}\right),
$$

where the $p_{\perp, i}$ and $\eta_{i}$ are defined with respect to the $q$ and $\bar{q}$ directions. Eq. (3.8) is valid even if $p_{\perp, 1} \sim p_{\perp, 2}$. The fundamental question that we ask is: do the parton showers reproduce this?

To examine this question we will ignore the subtlety that the Pythia kinematic $\left|p_{\perp}\right|$ can differ from the evolution $\left|p_{\perp \text {,evol }}\right|$, since this occurs with significant weight only in a rapidity region of $\mathcal{O}(1)$, which corresponds to a soft emission at wide angle. Therefore the remainder of the discussion in this section will apply equally well to the Pythia and Dire showers.

Let us follow the sequence of branchings that generates two emissions, concentrating on the tree-level aspects:

- One starts with a $\bar{q} q$ dipole. Then a value $v_{1}$ of the ordering variable is chosen, together with an associated $z_{1}, \phi_{1}$. This leads to an emission of a first gluon $g_{1}$, with transverse momentum $p_{\perp, 1}$ and rapidity $\eta_{1}$. One now has two dipoles: $\bar{q} g_{1}$ and $g_{1} q$.

- A value $v_{2}$ is chosen for the ordering variable, one selects one of the two current dipoles for branching, and within it one of its two ends. One then chooses the $z_{2}$ and $\phi_{2}$ splitting variables and generates a second gluon $g_{2}$. To understand the effective matrix element one should consider the sum over all four resulting situations. 
Let us first consider the four cases in the situation where $v_{2} \ll v_{1}$, which has the simplification that for the phase-space regions of interest, $\left|p_{\perp, i}\right|=v_{i}$. For convenience we will henceforth take $Q=1$.

1. $\bar{q} \rightarrow \bar{q} g_{2}$ splitting of the $\bar{q} g_{1}$ dipole, for which we adopt the shorthand $\bar{q}\left[g_{1}\right] \rightarrow \bar{q} g_{2}\left[g_{1}\right]$, putting the spectator parton of the dipole in square brackets. The region of phasespace that gets filled and the associated splitting weight are as follows (we remind the reader that emissions collinear to $\bar{q}$ have negative $\eta$ ):

$$
\ln v_{2} \ll \eta_{2} \ll \frac{1}{2}\left(\eta_{1}+\ln v_{1}\right) \quad \rightarrow \quad d \mathcal{P}_{2}=C_{F} \frac{2 \alpha_{s}\left(\left|p_{\perp, 2}^{2}\right|\right)}{\pi} d \eta_{2} \frac{d p_{\perp, 2}}{p_{\perp, 2}} .
$$

The notation $a \ll b$ for logarithmic variables like rapidities should be understood as meaning that $e^{a-b}$ is small. The left-hand bound on $\eta_{2}$ corresponds to the maximum allowed (negative) rapidity along the anti-quark direction, i.e. the hard-collinear limit for radiation from the $\bar{q}$. The right-hand bound is determined as the point of zero rapidity of the $\bar{q} g_{1}$ dipole in its own rest frame, but translated into the original $\bar{q} q$ rest frame. Both bounds are given to within corrections of $\mathcal{O}(1)$, which are irrelevant for the purpose of our discussion, since they generate subleading logarithmic corrections.

2. $g_{1}[\bar{q}] \rightarrow g_{1} g_{2}[\bar{q}]:$ part of this branching is collinear to gluon 1 (in the centre-of-mass frame of the hard scattering), and we ignore that part for now. The remainder is collinear either to the $\bar{q}$ or $q$, covering a rapidity region

$$
\frac{1}{2}\left(\eta_{1}+\ln v_{1}\right) \ll \eta_{2} \ll \eta_{1} \quad \rightarrow \quad d \mathcal{P}_{2}=\frac{C_{A}}{2} \frac{2 \alpha_{s}\left(\left|p_{\perp, 2}^{2}\right|\right)}{\pi} d \eta_{2} \frac{d p_{\perp, 2}}{p_{\perp, 2}} .
$$

This splitting weight $d \mathcal{P}_{2}$ here is identical to that in eq. (3.9), except for the replacement of $C_{F} \rightarrow C_{A} / 2$. This will be a source of problems: one thinks of the $g_{1} \rightarrow g_{1} g_{2}$ splitting as being the emission of a gluon from a gluon hence the $C_{A} / 2$ colour factor. However splitting the $\bar{q} g_{1}$ dipole into two equal parts in its rest frame causes some part of the radiation assigned to the gluonic part to be in a phase space region where it is closer in angle to the $\bar{q}$ or $q$ than it is to the gluon. In that region, the $C_{A} / 2$ colour factor is wrong.

3. $g_{1}[q] \rightarrow g_{1} g_{2}[q]$, which is analogous to eq. (3.10) but with $\bar{q} \rightarrow q$,

$$
\eta_{1} \ll \eta_{2} \ll \frac{1}{2}\left(\eta_{1}+\ln \frac{1}{v_{1}}\right) \quad \rightarrow \quad d \mathcal{P}_{2}=\frac{C_{A}}{2} \frac{2 \alpha_{s}\left(\left|p_{\perp, 2}^{2}\right|\right)}{\pi} d \eta_{2} \frac{d p_{\perp, 2}}{p_{\perp, 2}},
$$

and again with the erroneous $C_{A} / 2$ colour factor.

4. $q\left[g_{1}\right] \rightarrow q g_{2}\left[g_{1}\right]$ which is analogous to eq. (3.9),

$$
\frac{1}{2}\left(\eta_{1}+\ln \frac{1}{v_{1}}\right) \ll \eta_{2} \ll \ln \frac{1}{v_{2}} \rightarrow d \mathcal{P}_{2}=C_{F} \frac{2 \alpha_{s}\left(\left|p_{\perp, 2}^{2}\right|\right)}{\pi} d \eta_{2} \frac{d p_{\perp, 2}}{p_{\perp, 2}} .
$$




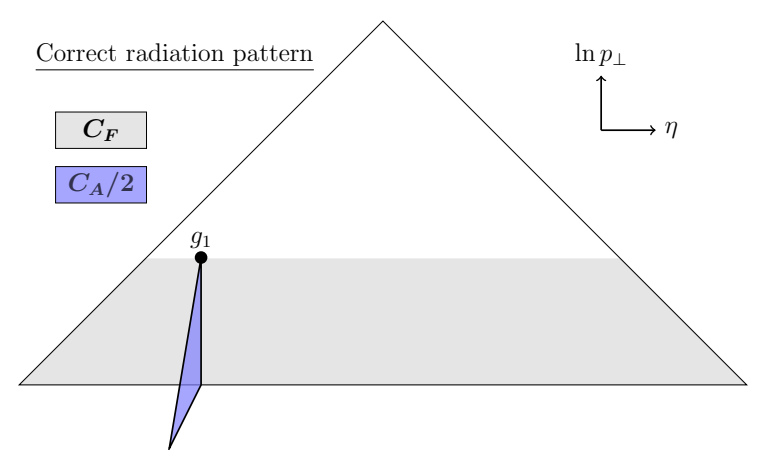

(a)

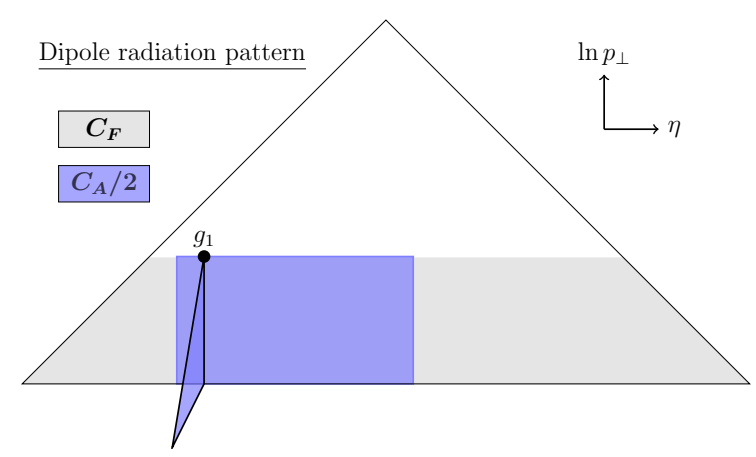

(b)

Figure 2. Lund-diagram illustrations of the subleading- $N_{C}$ issue in the showers that we consider. As a starting point we take a right (left)-moving quark (anti-quark), and gluon $g_{1}$ emitted at the $\eta-\ln p_{\perp}$ coordinate shown in the big ("primary") triangle. The phase-space for emission of a further gluon from the $q g_{1}$ dipole corresponds to the shaded area to the right of $g_{1}$ on the primary triangle, and the right-hand face of the "leaf" that comes out of the plane; analogously the phase-space for emission from the $\bar{q} g_{1}$ dipole corresponds to the shaded area of the primary triangle to the left of $g_{1}$ and to the left-hand face of the leaf. The colour factor associated with the phase-space region is indicated by the colour of the shading: grey denotes $C_{F}$, while blue denotes $C_{A} / 2$. The left-hand diagram shows the correct pattern, the right-hand diagram shows the outcome of the Pythia and Dire showers.

The main message to retain from this analysis is that there is a region that has both soft and collinear enhancements, for each of the two emissions, where instead of a $C_{F}^{2}$ colour factor, one obtains a $C_{F} C_{A} / 2$ colour factor, i.e. an incorrect subleading $N_{\mathrm{C}}$ term. This is illustrated in the Lund diagram of figure 2: panel (a) shows the correct assignment of colour factors across phase-space for radiation below the scale of $g_{1}$. The coloured "leaf" that comes out of the plane represents the additional phase-space that opens up following emission of $g_{1}$, with a $C_{A} / 2$ colour factor associated with each of its two faces. The restriction of the phase-space to that region is a consequence of angular ordering, as discussed for example some time ago in ref. [52]. Panel (b) shows the assignment that is effectively made in the case of the Pythia and Dire showers, with the coloured area $\left(C_{A} / 2\right)$ now extending into the primary Lund triangle. ${ }^{7}$ Since regions with simultaneous soft and collinear enhancements (i.e. extended areas in the Lund diagram) tend to be associated with leading double logarithms in distributions of common observables, one may expect that this issue with subleading $N_{\mathrm{C}}$ terms will also affect those double logarithms. We will investigate this in section 4.1.

We should note that issues with the attribution of colour factors beyond leading $N_{C}$ in dipole showers have been highlighted in a range of previous work, e.g. refs. [36, 53, 79, 80]. Our analysis in this subsection is close in particular to that of ref. [53]. We also note that approaches to obtain the correct subleading colour factor for at least the main soft-

\footnotetext{
${ }^{7}$ Note that since we start with a $q \bar{q}$ system, the primary plane emits only from the front face. For an initial $g g$ system, one might instead choose to represent emissions from both the front and rear faces, reflecting the presence of two $C_{A} / 2$ dipoles.
} 


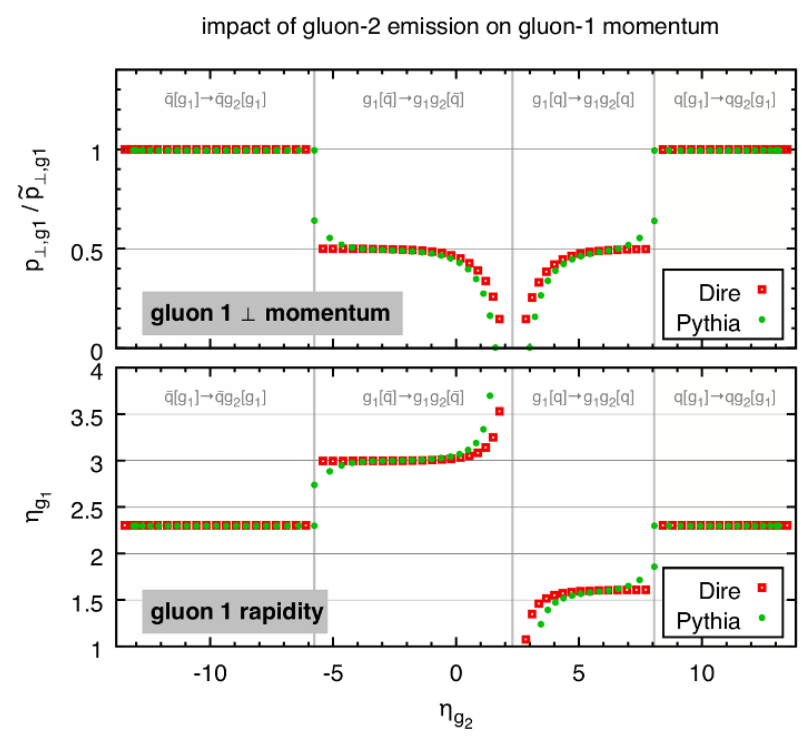

(a)

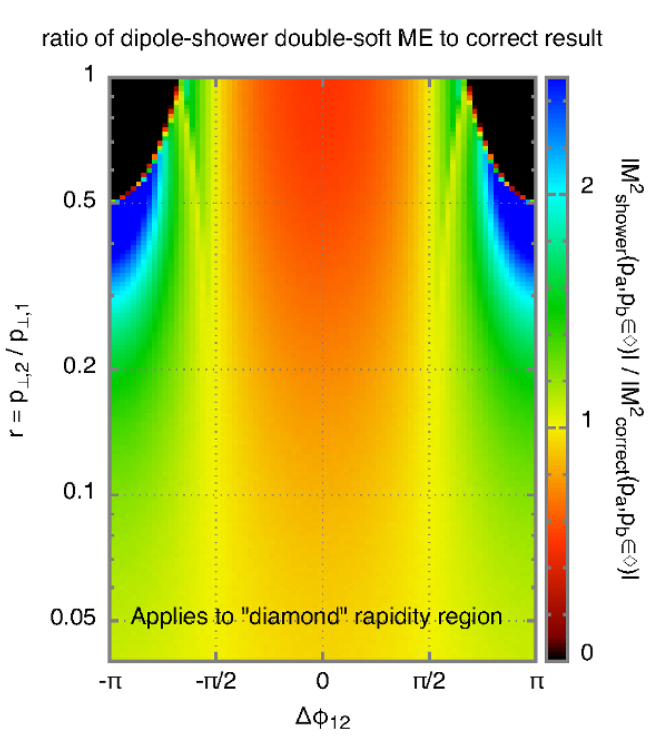

(b)

Figure 3. (a) Illustration of the modification of the transverse momentum (upper panel) and rapidity (lower panel) of gluon 1 after emission of gluon 2, shown as a function of the rapidity of gluon 2. Prior to emission of gluon 2 , gluon 1 originally has a rapidity $\eta_{g_{1}} \simeq 2.3$ and transverse momentum $\widetilde{p}_{\perp, g_{1}}=v_{1}=10^{-6} Q\left(v_{1}=10^{-6} Q\right.$ and $\left.1-z_{1}=10^{-5}\right)$. Gluon 2 has $v_{2}=\frac{1}{2} v_{1}$ and is emitted parallel in azimuth to gluon 1. To help guide the eye, four regions of gluon 2 rapidity are labelled according to the identity of the parton that branches and that of the spectator. The results have been obtained using a numerical implementation of the kinematic maps of section 2 . The transverse momentum shifts in (a) can be reinterpreted in terms of the effect they have on the effective matrix element for double-soft emission. Plot (b) shows the ratio of this effective matrix element to the true one, as a function of the azimuthal angle between the two emissions and their transverse-momentum ratio (in a specific "diamond" region of widely separated rapidities, cf. appendix A). For simplicity, the matrix-element ratio is given in the large- $N_{\mathrm{C}}$ limit.

collinear divergences have existed for some time. The classification that is implied by angular ordering (see also ref. [52]) provides a guide in this direction, as was articulated for a dipole shower in ref. [53] and found to be relevant for particle multiplicities at LHC energies [54]. Another proposal is that of ref. [79].

\subsection{Issues in two-emission case: single strong ordering}

Now we turn to the case where $v_{2}$ is only moderately smaller than $v_{1}$. Again one may consider the four cases listed in section 3.2, and in each case we will determine the kinematics of the four final-state partons. It is easiest to first illustrate what happens with reference to figure 3a. Here we have generated a sequence of two emissions, $g_{1}$ and $g_{2}$, with $v_{2}=v_{1} / 2$, and we study how the momentum of $g_{1}$ is modified after emission of $g_{2}$. Using $\widetilde{\boldsymbol{p}}_{\perp, g_{1}}$ and $\widetilde{\eta}_{g_{1}}\left(\boldsymbol{p}_{\perp, g_{1}}\right.$ and $\left.\eta_{g_{1}}\right)$ to denote the 2d-vector transverse momentum and rapidity respectively of gluon $g_{1}$ before (after) emission of $g_{2}$, the figure illustrates the following 
pattern of modifications:

$$
\begin{aligned}
& \text { 1. } \bar{q}\left[g_{1}\right] \rightarrow \bar{q} g_{2}\left[g_{1}\right]: \quad \boldsymbol{p}_{\perp, g_{1}}=\widetilde{\boldsymbol{p}}_{\perp, g_{1}}, \quad \eta_{g_{1}}=\widetilde{\eta}_{g_{1}}, \\
& \text { 2. } g_{1}[\bar{q}] \rightarrow g_{1} g_{2}[\bar{q}]: \quad \boldsymbol{p}_{\perp, g_{1}}=\widetilde{\boldsymbol{p}}_{\perp, g_{1}}-\boldsymbol{p}_{\perp, g_{2}}, \quad \eta_{g_{1}}=\widetilde{\eta}_{g_{1}}-\ln \frac{\left|\boldsymbol{p}_{\perp, g_{1}}\right|}{\left|\widetilde{\boldsymbol{p}}_{\perp, g_{1}}\right|}, \\
& \text { 3. } g_{1}[q] \rightarrow g_{1} g_{2}[q]: \quad \boldsymbol{p}_{\perp, g_{1}}=\widetilde{\boldsymbol{p}}_{\perp, g_{1}}-\boldsymbol{p}_{\perp, g_{2}}, \quad \eta_{g_{1}}=\widetilde{\eta}_{g_{1}}+\ln \frac{\left|\boldsymbol{p}_{\perp, g_{1}}\right|}{\left|\widetilde{\boldsymbol{p}}_{\perp, g_{1}}\right|}, \\
& \text { 4. } \quad q\left[g_{1}\right] \rightarrow q g_{2}\left[g_{1}\right]: \quad \boldsymbol{p}_{\perp, g_{1}}=\widetilde{\boldsymbol{p}}_{\perp, g_{1}}, \quad \eta_{g_{1}}=\widetilde{\eta}_{g_{1}}
\end{aligned}
$$

In regions 1 and 4, gluon 1 remains essentially unaffected by the emission of 2 (the transverse recoils are absorbed by the quark). This is correct, because in the exact matrix element, soft gluons that are widely separated in rapidity are independent of each other. In regions 2 and 3 , where $g_{2}$ is at relatively central rapidities, the situation is different: $g_{1}$ acquires a transverse recoil to balance the transverse momentum of $g_{2}$ : this causes the $p_{\perp, g_{1}} / \widetilde{p}_{\perp, g_{1}}$ to be equal to $\frac{1}{2}$ in the corresponding regions of figure 3 a. There is also a corresponding modification of the rapidity of $g_{1}$ and its sign and magnitude can be worked out by noting that the dipole mass must be conserved despite the modification of the transverse momentum of $g_{1}$, i.e. by imposing that $p_{\perp, g_{1}} e^{ \pm \eta_{g_{1}}}=\widetilde{p}_{\perp, g_{1}} e^{ \pm \widetilde{\eta}_{g_{1}}}$, where the choice of sign depends on the specific configuration.

These modifications of the transverse momentum and rapidity of gluon 1 after emission of a subsequent gluon 2 are a cause for concern. This is most easily seen by working out the effective splitting weight for the emission of two soft gluons in regions 2 and 3 . We concentrate on a specific "diamond" rapidity region, which has single-logarithmic rapidity enhancements for each of the gluons, and whose size is $1 / 3$ of the total double rapidity phase-space. The details and analysis are given in appendix A, and we concentrate here on the results. The result for the ratio of the effective matrix element to the correct one, eq. (3.8), is shown in figure $3 \mathrm{~b}$ as a function of the azimuthal angle between the two emissions and their transverse-momentum ratio. The figure reveals some unwanted features. These include the empty zones for $p_{\perp, 2} / p_{\perp, 1} \gtrsim \frac{1}{2}$ and $\left|\Delta \phi_{12}\right| \gtrsim 2 \pi / 3$ and the strong enhancement in a similar azimuthal region for $\frac{1}{4} \lesssim p_{\perp, 2} / p_{\perp, 1} \lesssim \frac{1}{2}$. There is also depletion and enhancement in other areas of the plot. Only for rather small values of $p_{\perp, 2} / p_{\perp, 1}$ does the effective shower matrix element tend to the correct result.

Some of the features of figure $3 \mathrm{~b}$ are straightforward to understand qualitatively. Consider, for example, the case when the second gluon is emitted back-to-back with respect to the first, $\Delta \phi_{12}=\pi$ and with a $p_{\perp, 2}$ that is a fraction $\widetilde{r}$ of the first emission's original $\widetilde{p}_{\perp, 1}$. The first emission's transverse momentum gets increased by a factor of $1+\widetilde{r}$, so that the new ratio of transverse momenta becomes $r=\widetilde{r} /(1+\widetilde{r})$. Since $\widetilde{r} \leq 1$, the final ratio $r$ is bounded to be less than $r \leq 1 / 2$. This generates the dead zone for $r>\frac{1}{2}$ and the strong enhancement just below.

As we will see below, the underlying recoil issue that leads to the incorrect double-soft tree-level matrix element will also cause many common observables, e.g. $e^{+} e^{-}$event shapes, to have incorrect NLL (leading- $N_{\mathrm{C}}$ ) terms in distributions as evaluated with parton showers.

The question of recoil in showers with dipole-local recoil was first raised long ago [51]. That analysis compared the effective shower matrix element to the full double-emission 
matrix element in $e^{+} e^{-}$collisions. In particular it highlighted the dead-zone problem that is visible near $\Delta \phi_{12}= \pm \pi$ and $r \simeq 1$ in our figure $3 \mathrm{~b}$. However, it differs from our analysis in that it did not take the formal soft and collinear limits, and as such could also not extend to the logarithmically-relevant limit of widely separated rapidities for the two emissions.

More recently, Nagy and Soper (e.g. refs. [81, 82]) and also Gieseke and Plätzer [62] (journal version) have highlighted issues to do with recoil in the context of the DrellYan (DY) transverse momentum distribution. The final-state recoil issue that we have discussed here is intimately connected with those recoil issues in the initial state. The novelty of our finding is that this type of issue applies not just to a specific observable, and not just to processes with initial-state hadrons, but to the full pattern of soft gluon radiation in essentially any process. Aspects introduced by Nagy and Soper, in particular the combination of a time-like ordering variable and a modified (global) recoil scheme, can, we believe, be critical ingredients in addressing the recoil problem that we are discussing. However the Nagy-Soper shower prescriptions are significantly more complicated than the Pythia or Dire showers and include a number of variants (e.g. both $p_{\perp}$ and time ordering). As we have seen in the Pythia single-emission case, e.g. issue 3 in section 3.1, subtleties can arise in almost any aspect of a shower, and a conclusion should only be drawn from a full, detailed analysis of a specific shower prescription.

A further point to be aware of is that any analysis of a proposed solution needs to go beyond the two-emission case. In particular, qualitatively new recoil-related issues can arise starting from the third emission. For example, the solution proposed in ref. [51] and adopted in the Ariadne program [50], is to assign recoil for a $q g$ dipole to the quark. While we believe this to be adequate at second order, the recoil issue reappears at 3rd order for emission from a $g g$ dipole, and is not, we believe, addressed by the solution of ref. [51].

\section{Logarithmic analysis at second order}

While section 3 illustrated physical shortcomings of two widespread showers, the key question that remains to answer is that of the consequences of those shortcomings. Insofar as a parton shower is supposed to provide resummation of logarithms, the natural way of examining those consequences is in terms of the impact on the logarithmic accuracy of the shower predictions for various classes of observable.

We will discuss the logarithmic accuracy in the context of event-shape variables, which have been widely studied and are well understood from the point of analytic and seminumerical resummation. Let $V$ be some event shape variable, a function of all the momenta in an event. The quantity we will study is $\Sigma(L)$, the probability that the event shape has a value smaller than $e^{-L}$. For most event-shape variables the structure of $\Sigma(L)$ is of the form

$$
\Sigma(L)=\exp \left[L g_{1}\left(\alpha_{s} L\right)+g_{2}\left(\alpha_{s} L\right)+\alpha_{s} g_{3}\left(\alpha_{s} L\right)+\cdots\right]+\mathcal{O}\left(\alpha_{s} e^{-L}\right),
$$

where we emphasise the logarithmically enhanced part of the result. In our default counting of logarithmic accuracy, the $\ln \Sigma$ counting, the $g_{1}\left(\alpha_{s} L\right)$ function contains the LL terms in $\ln \Sigma, \alpha_{s}^{n} L^{n+1}$; the $g_{2}\left(\alpha_{s} L\right)$ function contains the NLL terms, $\alpha_{s}^{n} L^{n}$; and so forth. Results up to NLL can be obtained for arbitrary global event shape type observables using the 
CAESAR formalism [55]. The formalism classifies observables in terms of two parameters, $a$ and $b$ according to their dependence on the momentum of a single soft-collinear emission

$$
V(p,\{\text { Born momenta }\}) \propto p_{\perp}^{a} e^{-\left|\eta_{p}\right| b},
$$

a classification that we will refer to below. The use of $\alpha_{s}^{\text {CMW }}$, cf. eqs. (2.20a)-(2.15) is sometimes held to be sufficient to reproduce NLL accuracy. While it is a necessary ingredient, together with two-loop running of the coupling, on their own these elements are not sufficient. Event shape observables are interesting to consider, with a view to future parton shower developments, because in many cases their resummation is known to NNLL or even higher accuracy [56, 83-87].

There is also an alternative, $\Sigma$ counting, reflecting a structure

$$
\Sigma(L)=\sum_{m=0}^{\infty} \sum_{n=0}^{2 m} h_{m, n} \alpha_{s}^{m} L^{n}+\mathcal{O}\left(\alpha_{s} e^{-L}\right) .
$$

In this counting, $\mathrm{LL}_{\Sigma}$ terms correspond to the $\alpha_{s}^{n} L^{2 n}$ contributions, $\mathrm{NLL}_{\Sigma}$ to $\alpha_{s}^{n} L^{2 n-1}$ and so forth. There are some instructive observables for which only eq. (4.3) can be used. These include $n$-jet rates in $e^{+} e^{-}$for $n \geq 3$ with Cambridge [88] and Durham [89] jet clustering. ${ }^{8}$ The $n$-jet rates are interesting also because the same physics that is required for their correctness enters into the calculation of the scaling of hadron multiplicities with energy [57].

\subsection{Subleading- $N_{\mathrm{C}} \alpha_{s}^{2} L^{4}$ terms}

Observables will break up into two basic classes from the point of view of subleading- $N_{\mathrm{C}}$ $\alpha_{s}^{2} L^{4}$ terms: those with $b \neq 0$ and those with $b=0$ in eq. (4.2). Let us start with the example of the thrust [91], i.e. a case with $a=1, b=1$. The $L L_{\Sigma}$ result for the thrust is

$$
\Sigma(L)=\exp \left(-\frac{\bar{\alpha} L^{2}}{2}\right)=1-\frac{\bar{\alpha} L^{2}}{2}+\frac{\bar{\alpha}^{2} L^{4}}{8}+\mathcal{O}\left(\bar{\alpha}^{3}\right)
$$

where

$$
\bar{\alpha}=\frac{2 \alpha_{s} C_{F}}{\pi} .
$$

To illustrate how the colour-factor issue of eqs. (3.10) and (3.11) impacts the doublelogarithmic structure, it is useful to consider figure 4. For a given constraint on the thrust, one has the thick red boundary: emissions above that boundary are vetoed. However there can be emissions below that boundary, e.g. emission $g_{1}$, which modify the colour factor for subsequent emissions (and their associated virtual corrections) with a lower value of the ordering variable $v$, but that are above the thrust boundary: instead of being associated with the correct $C_{F}$ factor, they have a factor $C_{A} / 2$. The region where this occurs is shown in blue, and corresponds to the rapidity regions of eqs. (3.10) and (3.11), cf. also figure 2b. The second-order, $\mathrm{LL}_{\Sigma}$ issue that arises because of this can be evaluated by considering the

\footnotetext{
${ }^{8}$ And also many non recursively infrared and collinear safe observables [55], such as the JADE jet rates [90].
} 


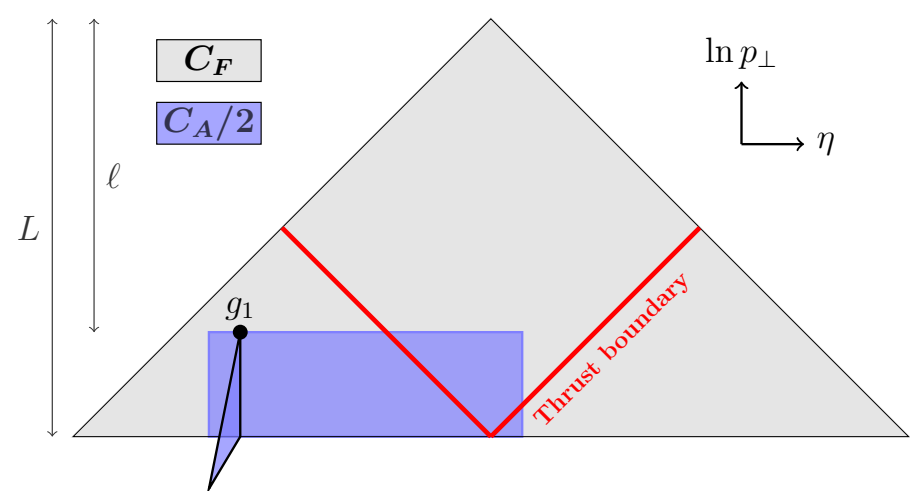

Figure 4. Lund diagram to help illustrate subleading- $N_{\mathrm{C}}$ issue for the Thrust. See text for further details.

area of the blue region that is above the thrust boundary, integrating also over the phase space for real emission 1 ,

$$
\begin{aligned}
\delta \Sigma(L) & =-2 \bar{\alpha}^{2} \int_{L / 2}^{L} d \ell_{1} \int_{-L+\ell_{1}}^{-\ell_{1}} d \eta_{1} \int_{\ell_{1}}^{L} d \ell_{2} \int_{\frac{1}{2}\left(\eta_{1}-\ell_{1}\right)}^{\frac{1}{2}\left(\eta_{1}+\ell_{1}\right)} d \eta_{2} \Theta\left(\left|\eta_{2}\right|<L-\ell_{2}\right)\left(\frac{C_{A}}{2 C_{F}}-1\right) \\
& =-\frac{1}{64} \bar{\alpha}^{2} L^{4}\left(\frac{C_{A}}{2 C_{F}}-1\right)
\end{aligned}
$$

where one should recall that $L$ is positive, we have introduced $\ell_{i}=\ln 1 / v_{i}, \bar{\alpha}$ includes a $C_{F}$ colour factor, cf. eq. (4.5), and there is an overall factor of two associated with the possibility of $\eta_{1}$ being either negative or positive (the integral includes only the negative case). This correction is double logarithmic. However it is $N_{\mathrm{C}}$ suppressed, by a factor

$$
c \equiv\left(\frac{C_{A}}{2 C_{F}}-1\right)=\frac{1}{N_{\mathrm{C}}^{2}-1}=\frac{1}{8} .
$$

A double-logarithmic $\left(\mathrm{LL}_{\Sigma}\right) N_{\mathrm{C}}$-suppressed effect of this kind is present for any eventshape like observable with $b \neq 0$ in eq. (4.2). The fact that terms $\alpha_{s}^{n} L^{2 n}$ are modified for $n \geq 2$ means that the result from the parton shower does not properly exponentiate beyond leading colour, i.e. cannot be written in the form of eq. (4.1).

In contrast, for observables with $b=0$, for example the jet broadening, there is no such effect at double-logarithmic accuracy. This is because the boundary associated with a limit on the value of a $b=0$ observable corresponds to a horizontal line in the Lund plane. As a result the only region in which it matters that an emission 1 modifies the colour for a subsequent emission 2 is when both emissions have a commensurate transverse momentum. This removes a number of logarithms and induces only a NLL $\mathrm{NL}_{\ln }$ type $N_{\mathrm{C}}$-suppressed effect.

We expect similar issues of a wrong subleading-colour coefficient for the double logarithms in the $e^{+} e^{-} 4$-jet (and higher) rates with the $k_{t}$ algorithm [89]. Note that these are somewhat different from the (leading-colour) issues discussed in ref. [92] for other, non-dipole classes of $p_{\perp}$ ordered shower. 


\subsection{Leading- $N_{\mathrm{C}} \alpha_{s}^{2} L^{2}$ terms}

If we work in the leading- $N_{\mathrm{C}}$ limit, $C_{A}=2 C_{F}$, then the impact of the incorrect shower mappings in regions 2 and 3 of eq. (3.13) can be written as follows (recall that we are using $Q=1$ )

$$
\begin{aligned}
\delta \Sigma(L)=\bar{\alpha}^{2} \int_{0}^{1} \frac{d v_{1}}{v_{1}} \int_{\ln v_{1}}^{\ln 1 / v_{1}} d \eta_{1} \int_{0}^{v_{1}} \frac{d v_{2}}{v_{2}} \int_{\frac{1}{2}\left(\eta_{1}+\ln v_{1}\right)}^{\frac{1}{2}\left(\eta_{1}+\ln 1 / v_{1}\right)} d \eta_{2} \int_{0}^{2 \pi} \frac{d \phi_{12}}{2 \pi} \times \\
\times\left[\Theta\left(e^{-L}-V\left(p_{1}^{\text {shower }}, p_{2}\right)\right)-\Theta\left(e^{-L}-V\left(p_{1}^{\text {correct }}, p_{2}\right)\right)\right]
\end{aligned}
$$

where we examine the difference between the double-real contribution with a "shower" mapping and a correct mapping. "Correct" means any mapping that leaves the transverse momentum and rapidity of $p_{1}$ unchanged for $\left|\eta_{1}-\eta_{2}\right| \gg 1$ and so reproduces the Abelian limit. Eq. (4.8) holds in the soft and collinear limit and for compactness in the arguments of $V$ we omit the momenta of the (hard) quark and anti-quark, keeping in mind that in any practical shower implementation they must of course be included. We do not need to consider virtual corrections because from the kinematic point of view any configuration with fewer than two emissions has the correct leading- $N_{\mathrm{C}}$ distribution of emitted partons and hence the virtual contribution cancels in the difference between "correct" and "shower" cases. We have omitted the $\phi_{1}$ azimuthal integral, and written the $\phi_{2}$ integral in terms of $\phi_{12}=\phi_{2}-\phi_{1}$. We work in a fixed-coupling limit, for simplicity.

To obtain a concrete result from eq. (4.8) we first consider the 2-jet rate in the Cambridge $e^{+} e^{-}$jet algorithm [88], which is akin to calculating the distribution of $V$ defined as $\sqrt{y_{\text {cut }}} .9$ The Cambridge algorithm has the simple property that $V\left(\left\{p_{i}\right\}\right)=\max _{i}\left\{p_{\perp, i}\right\}$ for soft collinear emissions that are widely separated in rapidity. This allows us to write

$$
V\left(p_{1}^{\text {correct }}, p_{2}\right)=v_{1} \quad V\left(p_{1}^{\text {shower }}, p_{2}\right)=\max \left(v_{2}, \sqrt{v_{1}^{2}+v_{2}^{2}-2 v_{1} v_{2} \cos \phi_{12}}\right) .
$$

The absence of dependence on the particle rapidities makes it straightforward to evaluate the $\eta_{1}$ and $\eta_{2}$ integrals, and it will also be convenient to introduce $\zeta=v_{2} / v_{1}$. We can then write

$$
\begin{aligned}
\delta \Sigma^{\operatorname{cam}}(L)= & \bar{\alpha}^{2} \int_{0}^{1} \frac{d v_{1}}{v_{1}} 2 \ln ^{2} \frac{1}{v_{1}} \int_{0}^{1} \frac{d \zeta}{\zeta} \int_{0}^{2 \pi} \frac{d \phi_{12}}{2 \pi} \times \\
& \times\left[\Theta\left(e^{-L}-v_{1} \max \left(\zeta, \sqrt{1+\zeta^{2}-2 \zeta \cos \phi_{12}}\right)\right)-\Theta\left(e^{-L}-v_{1}\right)\right] .
\end{aligned}
$$

This reduces to

$$
\begin{aligned}
\delta \Sigma^{\mathrm{cam}}(L) & =2 \bar{\alpha}^{2} L^{2} \int_{0}^{1} \frac{d \zeta}{\zeta} \int_{0}^{2 \pi} \frac{d \phi_{12}}{2 \pi} \ln \frac{1}{\max \left(\zeta, \sqrt{1+\zeta^{2}-2 \zeta \cos \phi_{12}}\right)}+\mathcal{O}\left(\bar{\alpha}^{2} L\right) \\
& =-0.18277 \bar{\alpha}^{2} L^{2}+\mathcal{O}\left(\bar{\alpha}^{2} L\right)
\end{aligned}
$$

This demonstrates the presence of a NLL deficiency that starts at order $\alpha_{s}^{2}$.

\footnotetext{
${ }^{9}$ It is also closely related to jet-veto survival factors at hadron colliders.
} 
Another simple observable is the fractional moment of the energy-energy correlation, $\mathrm{FC}_{1}$, defined in appendix I.2 of ref. [55], which reduces to $V\left(\left\{p_{i}\right\}\right)=\sum_{i} p_{\perp, i}$ (i.e. a scalar sum) in the soft-collinear limit, giving

$$
\begin{aligned}
\delta \Sigma^{\mathrm{FC}_{1}}(L) & =2 \bar{\alpha}^{2} L^{2} \int_{0}^{1} \frac{d \zeta}{\zeta} \int_{0}^{2 \pi} \frac{d \phi_{12}}{2 \pi} \ln \frac{1+\zeta}{\sqrt{1+\zeta^{2}-2 \zeta \cos \phi_{12}}+\zeta}+\mathcal{O}\left(\bar{\alpha}^{2} L\right) \\
& =-0.066934 \bar{\alpha}^{2} L^{2}+\mathcal{O}\left(\bar{\alpha}^{2} L\right) .
\end{aligned}
$$

The numerical coefficients in eqs. (4.11), (4.12) are not particularly large. Nevertheless they can be relevant, especially from the perspective of trying to obtain accurate parton showers for the LHC. Consider high- $p_{\perp}$ jets of a few $\mathrm{TeV}$, where one might probe the substructure using shapes such $N$-subjettiness ratios [93]. If one is sensitive to radiation at the $5 \mathrm{GeV}$ scale where $\alpha_{s} \simeq 0.2$, one finds $\bar{\alpha} L \simeq 1$ and so eq. (4.11) would point to effects of the order of $20 \%$. Another point of comparison is to the effect of the CMW correction (cf. eq. (2.15)), which for both these observables reads $\bar{\alpha} L^{2} \times\left(\alpha_{s} / 2 \pi\right) K=\bar{\alpha}^{2} L^{2} K /\left(4 C_{F}\right) \simeq 0.65 \bar{\alpha}^{2} L^{2}$. In a context where groups are seeking to develop showers with higher-accuracy splitting kernels [39, 41-44], phenomenologically such an effect should not be neglected.

Interestingly there are also observables for which the $\alpha_{s}^{2} L^{2}$ coefficient is zero. Perhaps the most notable is anything that relates to a vector sum over the emissions' transverse momenta. Keeping in mind that $\boldsymbol{p}_{\perp, 1}^{\text {shower }}=\widetilde{\boldsymbol{p}}_{\perp, 1}-\boldsymbol{p}_{\perp, 2}$, the shower vector sum, $\boldsymbol{p}_{\perp, 1}^{\text {shower }}+\boldsymbol{p}_{\perp, 2}$ is simply equal to $\left|\widetilde{\boldsymbol{p}}_{\perp, 1}\right|=v_{1}$, while the correct result is $v_{1} \sqrt{1+\zeta^{2}+2 \zeta \cos \phi_{12}}$. Since the following integral vanishes

$$
\int_{0}^{2 \pi} \frac{d \phi_{12}}{2 \pi} \ln \left(1+\zeta^{2}+2 \zeta \cos \phi_{12}\right)=0 \quad \text { for } 0<\zeta<1,
$$

there will be no $\alpha_{s}^{2} L^{2}$ error for any observable that reduces to such a vector sum. Vector-sum type observables and deficiencies of transverse-momentum showers with dipole-local recoil schemes have seen some discussion for initial-state showering. Nagy and Soper [81] noted that it could affect logarithmic accuracy, though we are not aware of a specific statement detailing what accuracy would be affected. An explicit study of local versus global recoil schemes in appendix $\mathrm{C}$ of the Dire paper [31] suggested that the numerical impact is small. This would not be surprising if our analysis here carries over to the initial-state case and implies a zero $\alpha_{s}^{2} L^{2}$ coefficient there too. Note that an $\alpha_{s}^{3} L^{3}$ study that we have carried out shows that the zero is not an all-order property. ${ }^{10}$

We have analysed two further observables that are somewhat more involved: the total jet broadening [95] has a non-zero $\alpha_{s}^{2} L^{2}$ coefficient, while the thrust is zero at $\alpha_{s}^{2} L^{2}$ but not zero at $\alpha_{s}^{3} L^{3}$ (an all-order analysis reveals further subtleties, however). The situation is summarised in table 1.

\footnotetext{
${ }^{10}$ For example, for an observable that reduces to the vector sum of the transverse momenta of all soft emissions in the two hemispheres of an $e^{+} e^{-}$event, there is an erroneous NLL contribution to $\Sigma(L)$ which starts with a term $\simeq-0.349 \bar{\alpha}^{3} L^{3}$. Such an observable is similar to the transverse momentum of the $Z$ boson in hadron-hadron collisions. For $e^{+} e^{-}$collisions we are not aware that such an observable has ever been explicitly studied, however we believe it should be possible to construct it, for example starting from the observation in appendix I.1 of ref. [55], that certain Berger-Kucs-Sterman angularities [94] effectively reduce to hemisphere vector sums.
} 


\begin{tabular}{|cc|}
\hline Observable & NLL $_{\ln \Sigma}$ discrepancy \\
\hline $1-T$ & $0.116_{-0.004}^{+0.004} \bar{\alpha}^{3} L^{3}$ \\
\hline vector $p_{t}$ sum & $-0.349_{-0.003}^{+0.003} \bar{\alpha}^{3} L^{3}$ \\
\hline$B_{T}$ & $-0.0167335 \bar{\alpha}^{2} L^{2}$ \\
\hline$y_{3}^{\text {cam }}$ & $-0.18277 \bar{\alpha}^{2} L^{2}$ \\
\hline $\mathrm{FC}_{1}$ & $-0.066934 \bar{\alpha}^{2} L^{2}$ \\
\hline
\end{tabular}

Table 1. The table summarises the NLL difference between the Pythia and Dire shower-algorithm results and the analytic resummation for different observables, at the first non-trivial perturbative order in $\bar{\alpha} L=2 C_{F} \alpha_{s} L / \pi$. The quoted uncertainty in the results, whenever present, corresponds to the statistical integration error.

\section{Conclusions}

In this article we have set out some of the formalism needed to address the question of the multi-scale accuracy of parton showers, specifically in the context of the Pythia and Dire transverse-momentum ordered showers. Our conclusions apply to both.

The showers essentially demonstrate all required conditions when considering a single emission, i.e. the first emission is generated in a way that reproduces the correct soft and collinear limits of the single-emission matrix element, including single logarithmic regions (i.e. large-angle soft and hard collinear splitting). However, the pattern of multiple emission that they generate has flaws in singular regions that are arguably serious. First we have found that there are double logarithmic regions, already from two emissions, where the matrix element is incorrect at subleading $N_{\mathrm{C}}$. This causes the subleading $N_{\mathrm{C}}$ terms of the leading double logarithms $\left(\mathrm{LL}_{\Sigma}\right)$ to be wrong for a number of simple and widely used observables, such as the thrust (and $n \geq 3$ jet rates). ${ }^{11}$

At leading- $N_{\mathrm{C}}$, we have found that the effective double-emission matrix element is wrong in a region where the two emissions have commensurate transverse momenta and disparate angles. This is illustrated in figure $3 \mathrm{~b}$ showing that there are logarithmically enhanced regions where the discrepancies are at the $100 \%$ level. This finding should perhaps not be surprising given the matrix-element versus shower comparisons performed long ago by the Lund group [51] for transverse-momentum ordered showers with dipole-local recoil.

\footnotetext{
${ }^{11}$ For some practitioners the surprise might be that there do exist observables, such as the jet broadenings and 2-jet rate, for which the $L_{\Sigma}$ answer is correct including its subleading- $N_{\mathrm{C}}$ terms. We believe that at double logarithmic level, the subleading colour issue is relatively straightforward to fix. One option is to appropriately split each dipole into regions of $C_{F}$ and $C_{A} / 2$, possibly with continuous transitions between them. The assignment would simply follow the parton identification that is used in angular ordered showers such as Herwig or equivalently outlined in ref. [52]. Note however that such an approach has a bookkeeping cost within any shower that at leading $N_{C}$ uses colour dipoles. In particular, any dipole, whether $q \bar{q}, q g$ or $g g$ can in general have an arbitrarily large number of $C_{F}$ and $C_{A} / 2$ regions, in an alternating sequence. This is not necessarily the only approach that one can envisage. Indeed other approaches have been proposed in refs. $[53,79]$. We prefer therefore not to advocate one or other fix for the subleading-colour issue without detailed studies of performance and computational complexity.
} 
The underlying characteristic in the shower algorithm that causes this, namely the specific attribution of recoil, leads to the $\mathrm{NLL}_{\ln \Sigma}$ terms being wrong for a wide range of eventshape like observables (independently of any aspects related to the CMW scheme for the strong coupling), though the coefficient of the error is modest. Given the broad similarities in choices made by other $p_{\perp}$-ordered dipole-type and antenna showers with local recoil, it would not be surprising if similar conclusions apply to those as well. The analysis methods that we have developed here provide some first elements of a set of tools for parton-shower authors to analyse and understand the logarithmic properties of their algorithms.

Our observations have a number of implications. 1) NLL discrepancies (whether those observed here for two specific transverse-momentum ordered showers, or those discussed in ref. [76] for angular-ordered showers) have the potential to affect prospects for precision physics in many of the experimental measurements that rely significantly on parton showers. 2) The large discrepancy in the two-emission matrix elements for the transversemomentum ordered showers studied here, may matter also in the field of jet substructure, where large gains in signal to background discrimination rely on the ability to exploit the pattern of correlations between emissions, notably with the help of machine learning. 3) Certain methods for matching parton showers with fixed-order calculations are made significantly more difficult if the singularity structure of matrix elements is incorrect. This would notably be the case for any extension of the MC@NLO method to NNLO. 4) Efforts to improve parton showers with higher-order splitting kernels would probably be most appropriately pursued within a framework that is free of the issues that we encountered here. In particular, while we discussed problems that arise for two emissions, the underlying causes of those problems will affect matrix elements for any number of emissions. The inclusion of higher-order corrections to splitting functions and of double-soft emission matrix elements might, we imagine, at best postpone the first order in $\alpha_{s}$ at which the all-order logarithmic issues first manifest themselves, but this remains a question that deserves further study.

Overall, the approach we have introduced here provides some of the insight needed to address the problem of how to systematically go about creating parton shower algorithms with controlled multi-scale accuracy.

\section{Acknowledgments}

We are grateful to Stefan Höche, Paolo Nason, Torbjörn Sjöstrand, Peter Skands, Gregory Soyez, Bryan Webber and Giulia Zanderighi for helpful discussions and comments on the manuscript. We also thank a referee for bringing references [51, 53, 54] to our attention and for helpful comments. The work of P.F.M. has been supported by a Marie Skłodowska Curie Individual Fellowship of the European Commission's Horizon 2020 Programme under contract number 702610 Resummation4PS. F.D. is supported by the SNF grant P2SKP2_165039 and by the Office of High Energy Physics of the U.S. Department of Energy (DOE) under grant DE-SC-0012567. K.H. was supported by the European Commission through the ERC Consolidator Grant HICCUP (No. 614577). K.H. also thanks the Science and Technology Facilities Council (STFC) for support via grant award ST/P000274/1. M.D. thanks the STFC for support via grant award ST/P000800/1 and the 


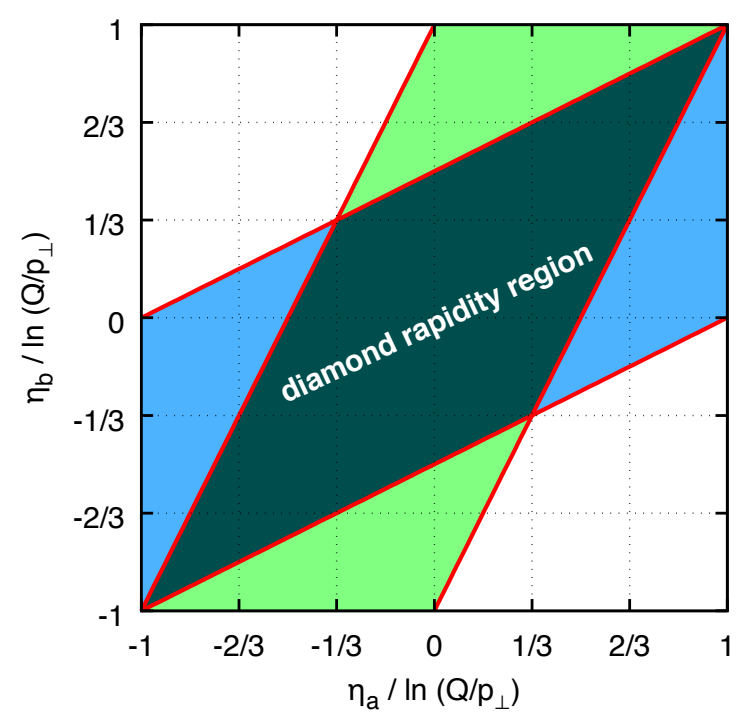

Figure 5. Representation of the different regions of rapidities for a pair of emissions with commensurate but small $p_{\perp}$ values. The "diamond" rapidity region is that used for the analysis in this appendix and also in figure $3 \mathrm{~b}$.

CERN theoretical physics department for a scientific associateship and for hospitality during the course of this work. M.D. also acknowledges the University of Manchester's School of Physics and Astronomy for sabbatical leave which facilitated this work. G.P.S. and P.F.M would like to thank the Munich Institute for Astronomy and Particle Physics (MIAPP) for hospitality and support during the Automated, Resummed and Effective programme.

\section{A Evaluation of double-soft effective matrix element}

There are several rapidity regions that can be considered when evaluating the double-soft effective matrix element. Let us consider a situation where $\ln p_{\perp, 1} / p_{\perp, 2} \sim \mathcal{O}(1)$ and where $\ln Q / p_{\perp, 1} \gg 1$. From the point of view of the identification of different rapidity regions, we will allow ourselves inaccuracies on the rapidity of $\mathcal{O}(1)$. In particular we will consider ratios such as $\eta / \ln \left(Q / p_{\perp}\right)$, where it is immaterial whether $p_{\perp}$ is $p_{\perp, 1}$ or $p_{\perp, 2}$.

Figure 5 shows the two dimensions associated with the rapidity variables, $\eta_{a}, \eta_{b}$ for the two emissions $a$ and $b$, each normalised to the maximum accessible rapidity, $\ln \left(Q / p_{\perp}\right)$. If emission $a$ comes first in the parton shower, then in the blue-shaded region,

$$
\frac{1}{2}\left(\eta_{a}-\ln \frac{Q}{p_{\perp}}\right)<\eta_{b}<\frac{1}{2}\left(\eta_{a}+\ln \frac{Q}{p_{\perp}}\right)
$$

emission $b$ will modify the transverse momentum of emission $a$. Conversely, if $b$ comes first in the parton shower, then in the green-shaded region,

$$
\frac{1}{2}\left(\eta_{b}-\ln \frac{Q}{p_{\perp}}\right)<\eta_{a}<\frac{1}{2}\left(\eta_{b}+\ln \frac{Q}{p_{\perp}}\right)
$$

emission $a$ will modify the transverse momentum of emission $b$. In the overlap, diamondshaped region, it is guaranteed that the second emission will always modify the transverse 
momentum of the first one, regardless of which of $a$ and $b$ was the first one. This is the region that we select for detailed analysis of the matrix element. Each of the blue and green shaded regions occupies half the plane and the diamond overlap region occupies $1 / 3$ of the plane. The region not affected at all by transverse recoil issues is also $1 / 3$ of the plane.

Recall that the correct double-soft matrix element is given by eq. (3.8). For the purpose of understanding the effective matrix element, we work in a fixed-coupling limit. Then consider the case where a shower generates first an emission $g_{1}$ with transverse momentum $\boldsymbol{p}_{\perp, 1}$ and rapidity $\eta_{1}$ and then an emission $g_{2}$ with transverse momentum $\boldsymbol{p}_{\perp, 2}$ and rapidity $\eta_{2}$, with the first emission's transverse momentum being modified by the recoil to become $\boldsymbol{p}_{\perp, 1}-\boldsymbol{p}_{\perp, 2}$. Then we evaluate the probability for either of $g_{1}$ and $g_{2}$ to coincide with some momentum $p_{a}$ and other one to coincide with some $p_{b}$. This is given by:

$$
\begin{aligned}
\frac{d P_{2, \text { shower }}\left(p_{a}, p_{b} \in \diamond\right)}{d \eta_{a} d \eta_{b} d^{2} \boldsymbol{p}_{\perp, a} d^{2} \boldsymbol{p}_{\perp, b}} & =\frac{1}{2 !}\left(\frac{\alpha_{s} C_{A}}{2 \pi^{2}}\right)^{2} \int \frac{d^{2} \boldsymbol{p}_{\perp, 1}}{p_{\perp, 1}{ }^{2}} \int_{p_{\perp, 2}<p_{\perp, 1}} \frac{d^{2} \boldsymbol{p}_{\perp, 2}}{p_{\perp, 2}{ }^{2}} \int_{\diamond} d \eta_{1} d \eta_{2} \times \\
\times & \left.\times \delta^{2}\left(\boldsymbol{p}_{\perp, 1}-\boldsymbol{p}_{\perp, 2}-\boldsymbol{p}_{\perp, a}\right) \delta^{2}\left(\boldsymbol{p}_{\perp, 2}-\boldsymbol{p}_{\perp, b}\right) \delta\left(\eta_{a}-\eta_{1}\right) \delta\left(\eta_{b}-\eta_{2}\right)+(a \leftrightarrow b)\right] .
\end{aligned}
$$

Note that we do not here consider the effect of the change of rapidity in eqs. (3.13), because the rapidity distributions of the gluons are uniform throughout most of the bulk of the diamond region and a shift in rapidity leaves those uniform distributions unchanged. The only exceptions are at the edge of the diamond region and along the diagonal (when the two rapidities are similar), and those regions' phase space is suppressed by one power of the logarithm of $v_{1} \simeq v_{2}$. Figure $3 \mathrm{~b}$ shows the ratio of eq. (A.3) to the correct result, eq. (3.8), in the large $N_{\mathrm{C}}$ limit, i.e. equating $C_{F}$ and $C_{A} / 2$.

\section{B Fixed-order difference with respect to NLL resummation}

In this appendix we report the necessary formulae to carry out the third-order study of the difference $\delta \Sigma(L)$ between the dipole showers considered in this work and the NLL analytic result, the results of which are summarised in table 1.

There are a number of simplifications that one can make in organising the calculation. A first simplification comes from the fact that the distribution of the first emission (prior to any of the subsequent emissions) is correctly described by the dipole showers considered here, and therefore the difference $\delta \Sigma(L)$ starts at $\mathcal{O}\left(\bar{\alpha}^{2}\right)$. Secondly, we are only interested in configurations in which the real emissions are simultaneously soft and collinear, and widely separated in rapidity from each other, which contribute to $\Sigma(L)$ starting at NLL. The correct emission probability in these configurations amounts to

$$
d P_{n}=\frac{C_{F}^{n}}{n !} \prod_{i=1}^{n}\left(\frac{2 \alpha_{s}\left(p_{\perp, i}^{2}\right)}{\pi} \frac{d p_{\perp, i}}{p_{\perp, i}} d \eta_{i} \frac{d \phi_{i}}{2 \pi}\right),
$$

where $p_{\perp, i}$ and $\eta_{i}$ are defined with respect to the $q$ and $\bar{q}$ directions and

$$
\left|\eta_{i}\right| \lesssim \ln \frac{Q}{p_{\perp, i}}
$$


Eq. (B.1) is valid as long as the emissions are very separated in rapidity, even if they have commensurate $p_{\perp, i}$.

A third observation is that, in the soft and collinear limit, the ordering variables of the dipole showers studied in this work coincide with the transverse momentum in eq. (B.1), hence we write $p_{\perp, i}=v_{i}$ in the following. Moreover, given that we are focusing on a fixed-order comparison, we can safely ignore running coupling effects and set

$$
C_{F} \frac{2 \alpha_{s}\left(p_{\perp}^{2}\right)}{\pi} \rightarrow C_{F} \frac{2 \alpha_{s}\left(Q^{2}\right)}{\pi}=\bar{\alpha} .
$$

We stress that all considerations made in this section are strictly valid in the large- $N_{c}$ limit, where we equate $C_{A}=2 C_{F}$.

We then start by considering a double-emission configuration, ordered in the transverse momenta $v_{i}$. In a shower picture, in addition to the real-emission probabilities, one needs to include the contribution of the no-emission probability between the hard scale $Q=1$ and the scale $v_{1}$ at which the first emission occurs, given by the following Sudakov form factor

$$
e^{-R\left(v_{1}\right)} \equiv \exp \left\{-\bar{\alpha} \int_{v_{1}}^{1} \frac{d p_{\perp}}{p_{\perp}} \int_{-\ln \frac{1}{p_{\perp}}}^{\ln \frac{1}{p_{\perp}}} d \eta \int_{0}^{2 \pi} \frac{d \phi}{2 \pi}\right\} .
$$

Equivalent suppression factors account for the no-emission probability between $v_{1}$ and $v_{2}$ and between $v_{2}$ and the shower cutoff, $v_{i}>Q_{0}$. One can expand the Sudakov factors out at fixed order and take the limit $Q_{0} \rightarrow 0$. This allows one to write all virtual corrections explicitly and obtain a fixed-order expansion of the shower equation.

Since the single-emission event is correctly described by the dipole showers, all single and double-virtual corrections at $\mathcal{O}\left(\bar{\alpha}^{2}\right)$ cancel (to NLL accuracy) in the difference $\delta \Sigma(L)$, which at this order is fully determined by the following double-real contribution

$$
\begin{aligned}
\delta \Sigma^{(2 \text { emissions })}(L)=\bar{\alpha}^{2} & \int_{0}^{1} \frac{d v_{1}}{v_{1}} \int_{\ln v_{1}}^{\ln 1 / v_{1}} d \eta_{1} \int_{0}^{v_{1}} \frac{d v_{2}}{v_{2}} \int_{\ln v_{2}}^{\ln 1 / v_{2}} d \eta_{2} \int_{0}^{2 \pi} \frac{d \phi_{1}}{2 \pi} \int_{0}^{2 \pi} \frac{d \phi_{2}}{2 \pi} \times \\
& \times\left[\Theta\left(e^{-L}-V\left(p_{1}^{\text {shower }}, p_{2}\right)\right)-\Theta\left(e^{-L}-V\left(p_{1}^{\text {correct }}, p_{2}\right)\right)\right],
\end{aligned}
$$

where we traded the $1 / 2$ ! multiplicity factor for the ordering $v_{1} \geq v_{2}$.

At the next non-trivial order we need to add configurations with three real emissions, for which one can repeat the above derivation obtaining

$$
\begin{aligned}
\delta \Sigma^{(3 \text { emissions })}(L)= & \bar{\alpha}^{3} \int_{0}^{1} \frac{d v_{1}}{v_{1}} \int_{0}^{v_{1}} \frac{d v_{2}}{v_{2}} \int_{0}^{v_{2}} \frac{d v_{3}}{v_{3}} \int_{\ln v_{1}}^{\ln 1 / v_{1}} d \eta_{1} \int_{\ln v_{2}}^{\ln 1 / v_{2}} d \eta_{2} \int_{\ln v_{3}}^{\ln 1 / v_{3}} d \eta_{3} \times \\
\times & \int_{0}^{2 \pi} \frac{d \phi_{1}}{2 \pi} \int_{0}^{2 \pi} \frac{d \phi_{2}}{2 \pi} \int_{0}^{2 \pi} \frac{d \phi_{3}}{2 \pi} \times \\
\times & {\left[\Theta\left(e^{-L}-V\left(p_{1}^{\text {shower }}, p_{2}^{\text {shower }}, p_{3}\right)\right)-\Theta\left(e^{-L}-V\left(p_{1}^{\text {correct }}, p_{2}^{\text {correct }}, p_{3}\right)\right)\right.} \\
& -\Theta\left(e^{-L}-V\left(p_{1}^{\text {shower }}, p_{2}\right)\right)+\Theta\left(e^{-L}-V\left(p_{1}^{\text {correct }}, p_{2}\right)\right) \\
& -\Theta\left(e^{-L}-V\left(p_{1}^{\text {shower }}, p_{3}\right)\right)+\Theta\left(e^{-L}-V\left(p_{1}^{\text {correct }}, p_{3}\right)\right) \\
& \left.-\Theta\left(e^{-L}-V\left(p_{2}^{\text {shower }}, p_{3}\right)\right)+\Theta\left(e^{-L}-V\left(p_{2}^{\text {correct }}, p_{3}\right)\right)\right] .
\end{aligned}
$$


The labels "correct" and "shower" in the momenta of eqs. (B.5) and (B.6) indicate that the emissions' momenta are modified according to the recoil prescription as either in the correct NLL result or in the dipole shower, respectively. While in the correct solution the recoil for the considered phase-space configurations is always absorbed by the emitting Born leg (either $q$ or $\bar{q}$ ), as discussed in section 3.3 this is not the case for the dipole showers analysed here. Instead, given $n-1$ emissions and the Born legs $q$ (along the positive $z$ direction) and $\bar{q}$ (along the negative $z$ direction), the recoil for the $n$-th emission $p_{n}$ can be assigned according to the following cases (as in the main text, we denote with a tilde all quantities prior to the emission of $p_{n}$ ):

- $p_{n}$ is emitted off the $\left[\bar{q} g_{i}\right]$ dipole (with $p_{i}$ being the momentum of the gluon $g_{i}$ colourconnected to the anti-quark $\bar{q})$ :

$$
\begin{aligned}
& \text { if } \eta_{n}>\frac{1}{2}\left(\ln \widetilde{p}_{\perp, i}+\widetilde{\eta}_{i}\right): \quad \boldsymbol{p}_{\perp, i}=\widetilde{\boldsymbol{p}}_{\perp, i}-\boldsymbol{p}_{\perp, n} ; \quad \eta_{i}=\widetilde{\eta}_{i}-\ln \frac{\left|\widetilde{\boldsymbol{p}}_{\perp, i}-\boldsymbol{p}_{\perp, n}\right|}{\left|\widetilde{\boldsymbol{p}}_{\perp, i}\right|} \\
& \text { if } \eta_{n}<\frac{1}{2}\left(\ln \widetilde{p}_{\perp, i}+\widetilde{\eta}_{i}\right): \quad \boldsymbol{p}_{\perp, \bar{q}}=\widetilde{\boldsymbol{p}}_{\perp, \bar{q}}-\boldsymbol{p}_{\perp, n} .
\end{aligned}
$$

- $p_{n}$ is emitted off the $\left[g_{i} q\right]$ dipole (with $p_{i}$ being the momentum of the gluon $g_{i}$ colourconnected to the quark $q$ ):

$$
\begin{aligned}
& \text { if } \eta_{n}<\frac{1}{2}\left(-\ln \widetilde{p}_{\perp, i}+\widetilde{\eta}_{i}\right): \quad \boldsymbol{p}_{\perp, i}=\widetilde{\boldsymbol{p}}_{\perp, i}-\boldsymbol{p}_{\perp, n} ; \quad \eta_{i}=\widetilde{\eta}_{i}+\ln \frac{\left|\widetilde{\boldsymbol{p}}_{\perp, i}-\boldsymbol{p}_{\perp, n}\right|}{\left|\widetilde{\boldsymbol{p}}_{\perp, i}\right|} \\
& \text { if } \eta_{n}>\frac{1}{2}\left(-\ln \widetilde{p}_{\perp, i}+\widetilde{\eta}_{i}\right): \quad \boldsymbol{p}_{\perp, q}=\widetilde{\boldsymbol{p}}_{\perp, q}-\boldsymbol{p}_{\perp, n} .
\end{aligned}
$$

- Finally, if $p_{n}$ is emitted off a $\left[g_{i} g_{j}\right]$ dipole (with $\widetilde{\eta}_{i}<\widetilde{\eta}_{j}$ ):

$$
\begin{aligned}
& \text { if } \eta_{n}<\frac{1}{2}\left(\ln \frac{\widetilde{p}_{\perp, j}}{\widetilde{p}_{\perp, i}}+\widetilde{\eta}_{i}+\widetilde{\eta}_{j}\right): \quad \boldsymbol{p}_{\perp, i}=\widetilde{\boldsymbol{p}}_{\perp, i}-\boldsymbol{p}_{\perp, n} ; \quad \eta_{i}=\widetilde{\eta}_{i}+\ln \frac{\left|\widetilde{\boldsymbol{p}}_{\perp, i}-\boldsymbol{p}_{\perp, n}\right|}{\left|\widetilde{\boldsymbol{p}}_{\perp, i}\right|}, \\
& \text { if } \eta_{n}>\frac{1}{2}\left(\ln \frac{\widetilde{p}_{\perp, j}}{\widetilde{p}_{\perp, i}}+\widetilde{\eta}_{i}+\widetilde{\eta}_{j}\right): \quad \boldsymbol{p}_{\perp, j}=\widetilde{\boldsymbol{p}}_{\perp, j}-\boldsymbol{p}_{\perp, n} ; \quad \eta_{j}=\widetilde{\eta}_{j}-\ln \frac{\left|\widetilde{\boldsymbol{p}}_{\perp, j}-\boldsymbol{p}_{\perp, n}\right|}{\left|\widetilde{\boldsymbol{p}}_{\perp, j}\right|} .
\end{aligned}
$$

In the virtual corrections corresponding to the $\Theta$ functions with only two emissions in eq. (B.6), the recoil procedure must be applied only to the momenta that are used in the corresponding observable, hence ignoring the third (virtual) momentum, which is insensitive to recoil.

The results obtained with the above formulae are reported in table 1 for a variety of observables. The evaluation of the $\mathcal{O}\left(\bar{\alpha}^{3}\right)$ coefficients is performed by computing eq. (B.6) numerically, and then fitting the coefficient of the leading term $\propto \bar{\alpha}^{3} L^{3}$. The result of this procedure is shown in figure 6 both for the thrust and for the vector sum of the emissions' transverse momenta, as defined in the main text. 


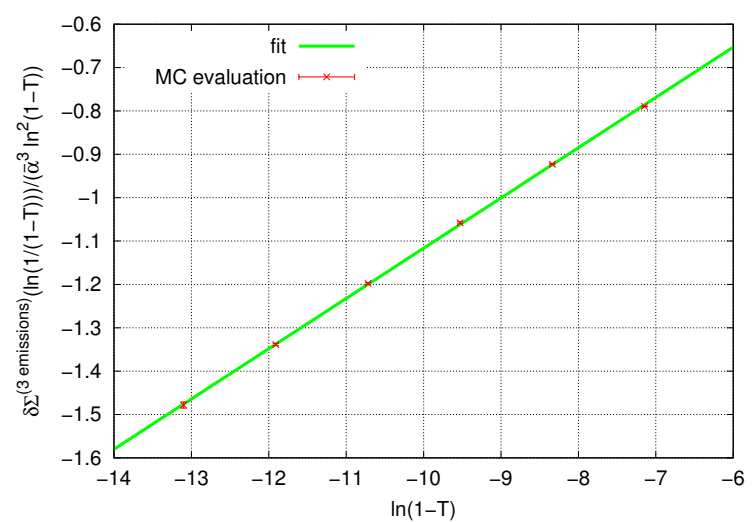

(a)

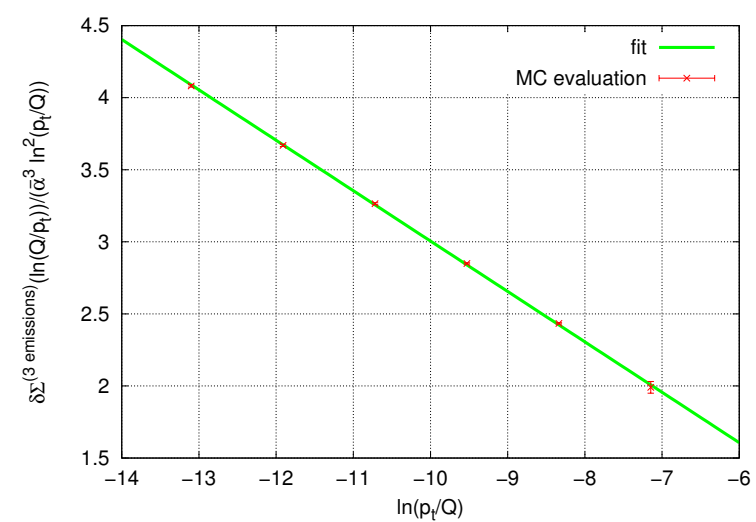

(b)

Figure 6. Difference between the third-order expansion of the dipole showers considered in this manuscript and the correct result obtained from analytic resummation at NLL, both for thrust (6a) and for the vectorial $p_{t}$ sum (6b). The data points correspond to the Monte Carlo result, divided by $\bar{\alpha}^{3} L^{2}$ so that a term proportional to $\bar{\alpha}^{3} L^{3}$ appears as a straight line, while the solid line is the resulting fit in the $L \in[-14,-6]$ range.

Open Access. This article is distributed under the terms of the Creative Commons Attribution License (CC-BY 4.0), which permits any use, distribution and reproduction in any medium, provided the original author(s) and source are credited.

\section{References}

[1] A. Buckley et al., General-purpose event generators for LHC physics, Phys. Rept. 504 (2011) 145 [arXiv:1101.2599] [INSPIRE].

[2] M.L. Mangano, M. Moretti and R. Pittau, Multijet matrix elements and shower evolution in hadronic collisions: $W b \bar{b}+n$ jets as a case study, Nucl. Phys. B 632 (2002) 343 [hep-ph/0108069] [INSPIRE].

[3] S. Catani, F. Krauss, R. Kuhn and B.R. Webber, QCD matrix elements + parton showers, JHEP 11 (2001) 063 [hep-ph/0109231] [INSPIRE].

[4] L. Lönnblad, Correcting the color dipole cascade model with fixed order matrix elements, JHEP 05 (2002) 046 [hep-ph/0112284] [INSPIRE].

[5] S. Frixione and B.R. Webber, Matching NLO QCD computations and parton shower simulations, JHEP 06 (2002) 029 [hep-ph/0204244] [INSPIRE].

[6] J. Alwall et al., The automated computation of tree-level and next-to-leading order differential cross sections and their matching to parton shower simulations, JHEP 07 (2014) 079 [arXiv: 1405.0301] [INSPIRE].

[7] P. Nason, A New method for combining NLO QCD with shower Monte Carlo algorithms, JHEP 11 (2004) 040 [hep-ph/0409146] [INSPIRE].

[8] S. Frixione, P. Nason and C. Oleari, Matching NLO QCD computations with Parton Shower simulations: the POWHEG method, JHEP 11 (2007) 070 [arXiv:0709.2092] [INSPIRE]. 
[9] S. Alioli, P. Nason, C. Oleari and E. Re, A general framework for implementing NLO calculations in shower Monte Carlo programs: the POWHEG BOX, JHEP 06 (2010) 043 [arXiv: 1002.2581] [INSPIRE].

[10] S. Jadach, W. Płaczek, S. Sapeta, A. Siódmok and M. Skrzypek, Matching NLO QCD with parton shower in Monte Carlo scheme - the KrkNLO method, JHEP 10 (2015) 052 [arXiv: 1503.06849] [INSPIRE].

[11] S. Hoeche, F. Krauss, M. Schonherr and F. Siegert, QCD matrix elements + parton showers: The NLO case, JHEP 04 (2013) 027 [arXiv: 1207.5030] [INSPIRE].

[12] K. Hamilton, P. Nason and G. Zanderighi, MINLO: Multi-Scale Improved NLO, JHEP 10 (2012) 155 [arXiv:1206.3572] [InSPIRE].

[13] R. Frederix and S. Frixione, Merging meets matching in MC@NLO, JHEP 12 (2012) 061 [arXiv: 1209.6215] [INSPIRE].

[14] K. Hamilton, P. Nason, E. Re and G. Zanderighi, NNLOPS simulation of Higgs boson production, JHEP 10 (2013) 222 [arXiv:1309.0017] [INSPIRE].

[15] S. Höche, Y. Li and S. Prestel, Drell-Yan lepton pair production at NNLO QCD with parton showers, Phys. Rev. D 91 (2015) 074015 [arXiv: 1405.3607] [INSPIRE].

[16] S. Alioli, C.W. Bauer, C. Berggren, F.J. Tackmann, J.R. Walsh and S. Zuberi, Matching Fully Differential NNLO Calculations and Parton Showers, JHEP 06 (2014) 089 [arXiv: 1311.0286] [INSPIRE].

[17] T. Sjöstrand and M. van Zijl, A Multiple Interaction Model for the Event Structure in Hadron Collisions, Phys. Rev. D 36 (1987) 2019 [INSPIRE].

[18] J.M. Butterworth, J.R. Forshaw and M.H. Seymour, Multiparton interactions in photoproduction at HERA, Z. Phys. C 72 (1996) 637 [hep-ph/9601371] [INSPIRE].

[19] I. Borozan and M.H. Seymour, An Eikonal model for multiparticle production in hadron hadron interactions, JHEP 09 (2002) 015 [hep-ph/0207283] [INSPIRE].

[20] T. Sjöstrand and P.Z. Skands, Multiple interactions and the structure of beam remnants, JHEP 03 (2004) 053 [hep-ph/0402078] [INSPIRE].

[21] T. Sjöstrand and P.Z. Skands, Transverse-momentum-ordered showers and interleaved multiple interactions, Eur. Phys. J. C 39 (2005) 129 [hep-ph/0408302] [INSPIRE].

[22] M. Bahr, S. Gieseke and M.H. Seymour, Simulation of multiple partonic interactions in HERWIG++, JHEP 07 (2008) 076 [arXiv:0803.3633] [INSPIRE].

[23] R. Corke and T. Sjöstrand, Multiparton Interactions and Rescattering, JHEP 01 (2010) 035 [arXiv: 0911.1909] [INSPIRE].

[24] R. Corke and T. Sjöstrand, Multiparton Interactions with an x-dependent Proton Size, JHEP 05 (2011) 009 [arXiv: 1101.5953] [INSPIRE].

[25] M.H. Seymour and A. Siodmok, Constraining MPI models using $\sigma_{\text {eff }}$ and recent Tevatron and LHC Underlying Event data, JHEP 10 (2013) 113 [arXiv:1307.5015] [INSPIRE].

[26] A. Buckley, H. Hoeth, H. Lacker, H. Schulz and J.E. von Seggern, Systematic event generator tuning for the LHC, Eur. Phys. J. C 65 (2010) 331 [arXiv:0907.2973] [INSPIRE].

[27] P.Z. Skands, Tuning Monte Carlo Generators: The Perugia Tunes, Phys. Rev. D 82 (2010) 074018 [arXiv: 1005 . 3457] [INSPIRE]. 
[28] A.J. Larkoski, I. Moult and B. Nachman, Jet Substructure at the Large Hadron Collider: A Review of Recent Advances in Theory and Machine Learning, arXiv:1709.04464 [INSPIRE].

[29] ATLAS collaboration, Jet energy measurement and its systematic uncertainty in proton-proton collisions at $\sqrt{s}=7 \mathrm{TeV}$ with the ATLAS detector, Eur. Phys. J. C 75 (2015) 17 [arXiv: 1406.0076] [INSPIRE].

[30] CMS collaboration, Jet energy scale and resolution in the CMS experiment in pp collisions at $8 \mathrm{TeV}, 2017$ JINST 12 P02014 [arXiv: 1607.03663] [INSPIRE].

[31] S. Höche and S. Prestel, The midpoint between dipole and parton showers, Eur. Phys. J. C 75 (2015) 461 [arXiv: 1506.05057] [INSPIRE].

[32] W.T. Giele, D.A. Kosower and P.Z. Skands, A simple shower and matching algorithm, Phys. Rev. D 78 (2008) 014026 [arXiv:0707.3652] [INSPIRE].

[33] N. Fischer, S. Prestel, M. Ritzmann and P. Skands, Vincia for Hadron Colliders, Eur. Phys. J. C 76 (2016) 589 [arXiv:1605.06142] [InSPIRE].

[34] Z. Nagy and D.E. Soper, A parton shower based on factorization of the quantum density matrix, JHEP 06 (2014) 097 [arXiv: 1401.6364] [INSPIRE].

[35] Z. Nagy and D.E. Soper, Parton showers with quantum interference, JHEP 09 (2007) 114 [arXiv: 0706.0017] [INSPIRE].

[36] Z. Nagy and D.E. Soper, Parton shower evolution with subleading color, JHEP 06 (2012) 044 [arXiv: 1202.4496] [INSPIRE].

[37] Z. Nagy and D.E. Soper, Effects of subleading color in a parton shower, JHEP 07 (2015) 119 [arXiv: 1501.00778] [INSPIRE].

[38] R. Ángeles Martínez, M. De Angelis, J.R. Forshaw, S. Plätzer and M.H. Seymour, Soft gluon evolution and non-global logarithms, arXiv:1802.08531 [INSPIRE].

[39] S. Jadach, A. Kusina, M. Skrzypek and M. Slawinska, Monte Carlo modelling of NLO DGLAP QCD evolution in the fully unintegrated form, Nucl. Phys. Proc. Suppl. 205-206 (2010) 295 [arXiv: 1007.2437] [INSPIRE].

[40] S. Jadach, A. Kusina, W. Placzek and M. Skrzypek, On the dependence of QCD splitting functions on the choice of the evolution variable, JHEP 08 (2016) 092 [arXiv:1606. 01238] [INSPIRE].

[41] S. Höche, F. Krauss and S. Prestel, Implementing NLO DGLAP evolution in Parton Showers, JHEP 10 (2017) 093 [arXiv:1705.00982] [inSPIRE].

[42] S. Höche and S. Prestel, Triple collinear emissions in parton showers, Phys. Rev. D 96 (2017) 074017 [arXiv: 1705.00742] [INSPIRE].

[43] F. Dulat, S. Höche and S. Prestel, Leading-Color Fully Differential Two-Loop Soft Corrections to QCD Dipole Showers, arXiv:1805.03757 [INSPIRE].

[44] H.T. Li and P. Skands, A framework for second-order parton showers, Phys. Lett. B 771 (2017) 59 [arXiv:1611.00013] [INSPIRE].

[45] A. Bassetto, M. Ciafaloni and G. Marchesini, Jet Structure and Infrared Sensitive Quantities in Perturbative QCD, Phys. Rept. 100 (1983) 201 [INSPIRE].

[46] Y.L. Dokshitzer, G. Marchesini and G. Oriani, Measuring color flows in hard processes: Beyond leading order, Nucl. Phys. B 387 (1992) 675 [INSPIRE]. 
[47] J.M. Campbell and E.W.N. Glover, Double unresolved approximations to multiparton scattering amplitudes, Nucl. Phys. B 527 (1998) 264 [hep-ph/9710255] [INSPIRE].

[48] S. Catani and M. Grazzini, Infrared factorization of tree level QCD amplitudes at the next-to-next-to-leading order and beyond, Nucl. Phys. B 570 (2000) 287 [hep-ph/9908523] [INSPIRE].

[49] G. Gustafson and U. Pettersson, Dipole Formulation of QCD Cascades, Nucl. Phys. B 306 (1988) 746 [INSPIRE].

[50] L. Lönnblad, ARIADNE version 4: A program for simulation of QCD cascades implementing the color dipole model, Comput. Phys. Commun. 71 (1992) 15 [InSPIRE].

[51] B. Andersson, G. Gustafson and C. Sjogren, Comparison of the dipole cascade model versus $O\left(\alpha_{s}^{2}\right)$ matrix elements and color interference in $e^{+} e^{-}$annihilation, Nucl. Phys. B 380 (1992) 391 [INSPIRE].

[52] G. Gustafson, Multiplicity distributions in QCD cascades, Nucl. Phys. B 392 (1993) 251 [INSPIRE].

[53] C. Friberg, G. Gustafson and J. Hakkinen, Color connections in $e^{+} e^{-}$annihilation, Nucl. Phys. B 490 (1997) 289 [hep-ph/9604347] [INSPIRE].

[54] P. Eden and G. Gustafson, Energy and virtuality scale dependence in quark and gluon jets, JHEP 09 (1998) 015 [hep-ph/9805228] [INSPIRE].

[55] A. Banfi, G.P. Salam and G. Zanderighi, Principles of general final-state resummation and automated implementation, JHEP 03 (2005) 073 [hep-ph/0407286] [INSPIRE].

[56] A. Banfi, H. McAslan, P.F. Monni and G. Zanderighi, A general method for the resummation of event-shape distributions in $e^{+} e^{-}$annihilation, JHEP 05 (2015) 102 [arXiv:1412.2126] [INSPIRE].

[57] G. Marchesini and B.R. Webber, Simulation of QCD Jets Including Soft Gluon Interference, Nucl. Phys. B 238 (1984) 1 [InSPIRE].

[58] T. Sjöstrand, S. Mrenna and P.Z. Skands, PYTHIA 6.4 Physics and Manual, JHEP 05 (2006) 026 [hep-ph/0603175] [inSPIRE].

[59] T. Sjöstrand et al., An Introduction to PYTHIA 8.2, Comput. Phys. Commun. 191 (2015) 159 [arXiv: 1410.3012] [INSPIRE].

[60] T. Gleisberg et al., Event generation with SHERPA 1.1, JHEP 02 (2009) 007 [arXiv:0811.4622] [INSPIRE].

[61] S. Schumann and F. Krauss, A Parton shower algorithm based on Catani-Seymour dipole factorisation, JHEP 03 (2008) 038 [arXiv:0709.1027] [INSPIRE].

[62] S. Platzer and S. Gieseke, Coherent Parton Showers with Local Recoils, JHEP 01 (2011) 024 [arXiv:0909.5593] [INSPIRE].

[63] S. Gieseke, P. Stephens and B. Webber, New formalism for QCD parton showers, JHEP 12 (2003) 045 [hep-ph/0310083] [inSPIRE].

[64] D. Reichelt, P. Richardson and A. Siodmok, Improving the Simulation of Quark and Gluon Jets with HERWIG 7, Eur. Phys. J. C 77 (2017) 876 [arXiv:1708.01491] [INSPIRE].

[65] J. Bellm et al., HERWIG \%.0/HERWIG++ 3.0 release note, Eur. Phys. J. C 76 (2016) 196 [arXiv: 1512.01178] [INSPIRE]. 
[66] S. Catani and M.H. Seymour, A general algorithm for calculating jet cross-sections in NLO QCD, Nucl. Phys. B 485 (1997) 291 [Erratum ibid. B 510 (1998) 503] [hep-ph/9605323] [INSPIRE].

[67] S. Catani, B.R. Webber and G. Marchesini, QCD coherent branching and semiinclusive processes at large $x$, Nucl. Phys. B 349 (1991) 635 [inSPIRE].

[68] V.N. Gribov and L.N. Lipatov, Deep inelastic e p scattering in perturbation theory, Sov. J. Nucl. Phys. 15 (1972) 438 [inSPIRE].

[69] G. Altarelli and G. Parisi, Asymptotic Freedom in Parton Language, Nucl. Phys. B 126 (1977) 298 [INSPIRE].

[70] Y.L. Dokshitzer, Calculation of the Structure Functions for Deep Inelastic Scattering and $e^{+} e^{-}$Annihilation by Perturbation Theory in Quantum Chromodynamics., Sov. Phys. JETP 46 (1977) 641 [INSPIRE].

[71] Yu.L. Dokshitzer and G. Marchesini, Monte Carlo and large angle gluon radiation, JHEP 03 (2009) 117 [arXiv:0809.1749] [INSPIRE].

[72] Z. Nagy and D.E. Soper, Final state dipole showers and the DGLAP equation, JHEP 05 (2009) 088 [arXiv:0901.3587] [INSPIRE].

[73] P.Z. Skands and S. Weinzierl, Some remarks on dipole showers and the DGLAP equation, Phys. Rev. D 79 (2009) 074021 [arXiv: 0903.2150] [INSPIRE].

[74] J.C. Collins, Spin Correlations in Monte Carlo Event Generators, Nucl. Phys. B 304 (1988) 794 [INSPIRE].

[75] I.G. Knowles, A Linear Algorithm for Calculating Spin Correlations in Hadronic Collisions, Comput. Phys. Commun. 58 (1990) 271 [INSPIRE].

[76] A. Banfi, G. Corcella and M. Dasgupta, Angular ordering and parton showers for non-global QCD observables, JHEP 03 (2007) 050 [hep-ph/0612282] [INSPIRE].

[77] Y. Hatta and T. Ueda, Resummation of non-global logarithms at finite $N_{c}$, Nucl. Phys. B 874 (2013) 808 [arXiv: 1304.6930] [INSPIRE].

[78] B. Andersson, G. Gustafson, L. Lönnblad and U. Pettersson, Coherence Effects in Deep Inelastic Scattering, Z. Phys. C 43 (1989) 625 [INSPIRE].

[79] W.T. Giele, D.A. Kosower and P.Z. Skands, Higher-Order Corrections to Timelike Jets, Phys. Rev. D 84 (2011) 054003 [arXiv: 1102.2126] [InSPIRE].

[80] L. Hartgring, E. Laenen and P. Skands, Antenna Showers with One-Loop Matrix Elements, JHEP 10 (2013) 127 [arXiv:1303.4974] [INSPIRE].

[81] Z. Nagy and D.E. Soper, On the transverse momentum in Z-boson production in a virtuality ordered parton shower, JHEP 03 (2010) 097 [arXiv:0912.4534] [INSPIRE].

[82] Z. Nagy and D.E. Soper, Ordering variable for parton showers, JHEP 06 (2014) 178 [arXiv: 1401.6366] [INSPIRE].

[83] T. Becher and M.D. Schwartz, A precise determination of $\alpha_{s}$ from LEP thrust data using effective field theory, JHEP 07 (2008) 034 [arXiv:0803.0342] [INSPIRE].

[84] P.F. Monni, T. Gehrmann and G. Luisoni, Two-Loop Soft Corrections and Resummation of the Thrust Distribution in the Dijet Region, JHEP 08 (2011) 010 [arXiv:1105.4560] [INSPIRE]. 
[85] A.H. Hoang, D.W. Kolodrubetz, V. Mateu and I.W. Stewart, $C$-parameter distribution at $N^{3} L L^{\prime}$ including power corrections, Phys. Rev. D 91 (2015) 094017 [arXiv:1411.6633] [INSPIRE].

[86] T. Becher and G. Bell, NNLL Resummation for Jet Broadening, JHEP 11 (2012) 126 [arXiv: 1210.0580] [INSPIRE].

[87] A. Banfi, H. McAslan, P.F. Monni and G. Zanderighi, The two-jet rate in $e^{+} e^{-}$at next-to-next-to-leading-logarithmic order, Phys. Rev. Lett. 117 (2016) 172001 [arXiv: 1607.03111] [INSPIRE].

[88] Y.L. Dokshitzer, G.D. Leder, S. Moretti and B.R. Webber, Better jet clustering algorithms, JHEP 08 (1997) 001 [hep-ph/9707323] [INSPIRE].

[89] S. Catani, Y.L. Dokshitzer, M. Olsson, G. Turnock and B.R. Webber, New clustering algorithm for multi-jet cross-sections in $e^{+} e^{-}$annihilation, Phys. Lett. B 269 (1991) 432 [INSPIRE].

[90] N. Brown and W.J. Stirling, Jet cross-sections at leading double logarithm in $e^{+} e^{-}$ annihilation, Phys. Lett. B 252 (1990) 657 [INSPIRE].

[91] E. Farhi, A QCD Test for Jets, Phys. Rev. Lett. 39 (1977) 1587 [inSPIRE].

[92] B.R. Webber, QCD Jets and Parton Showers, in Quantum chromodynamics and beyond: Gribov-80 memorial volume. Proceedings, Memorial Workshop devoted to the 80th birthday of V.N. Gribov, Trieste, Italy, May 26-28, 2010, pp. 82-92 (2011) [DOI:10.1142/9789814350198_0010] [arXiv: 1009.5871] [INSPIRE].

[93] J. Thaler and K. Van Tilburg, Identifying Boosted Objects with N-subjettiness, JHEP 03 (2011) 015 [arXiv: 1011.2268] [INSPIRE].

[94] C.F. Berger, T. Kucs and G.F. Sterman, Event shape/energy flow correlations, Phys. Rev. D 68 (2003) 014012 [hep-ph/0303051] [INSPIRE].

[95] S. Catani, G. Turnock and B.R. Webber, Jet broadening measures in $e^{+} e^{-}$annihilation, Phys. Lett. B 295 (1992) 269 [INSPIRE]. 